\title{
Investment Horizons and Asset Prices under Asymmetric Information *
}

\author{
Elias Albagli
}

December 4, 2012

\begin{abstract}
I construct a generalized OLG economy where investors live for an arbitrary number of periods, and trade an infinitely-lived risky asset. Investors are rational but asymmetrically informed about the value of future dividends. I then compare asset pricing moments, and the informational role of prices, across economies with different investment horizons. Horizons affects prices through two key mechanisms. As horizons increase, 1) the age-adjusted risk aversion of the average investor falls, and 2) the risk transfer from forced liquidators into voluntary buyers drops. These mechanisms allow equilibria that fail to exist for short horizons to be recovered for high enough lifespans. There are typically two equilibria: a stable, low-volatility equilibrium in which longer horizons reduce price variability and raise average prices, and an unstable, high-volatility equilibrium with the opposite properties. Along the stable equilibrium, the reduction in non-fundamental price volatility caused by longer lifespans incites more aggressive trading by the informed investors, which impound more of their knowledge into prices. Longer investment horizons thus improve market efficiency, and reduce the uncertainty of the uninformed investors. Expected returns and return volatility are similar to an economy with full-information about fundamentals, even if the informed are relatively few. For short horizons, cautious trading disaggregates information from prices, and the economy approaches one with no private information.
\end{abstract}

JEL codes: E23, E32, G12, G14, G23.

Keywords: Investment horizons, asymmetric information, asset prices.

\footnotetext{
* University of Southern California, Marshall FBE. F: 213-7406418. albagli@marshall.usc.edu.
} 


\section{Introduction}

The fact that investors care about returns over a limited horizon is a pervasive feature of financial markets. With trading carried out mostly by intermediaries who care about short-term performance -be it through explicit contracts, or by the threat of fleeing investors- one has good reasons to suspect long-run prospects might often be underweighted in everyday market transactions. From a more cyclical perspective, the outset of financial crises are characterized by widespread investors' withdrawals and fund liquidations, suggesting fund managers' bias towards immediacy might be particularly acute during such episodes. This opens the question of whether the radically different behavior of markets during crises -sharp price drops, heightened volatility, and higher expected returns- could be partly explained by variations in the effective horizons of intermediaries. Moreover, even leaving intermediation out of the story, the fact that households literally have finite lifespans suggests concerns about the short-term are relevant.

Despite the apparent importance of constructing models where finite horizons play an explicit role, our knowledge in this regard seems limited. Mainstream asset pricing theory often assumes infinitely-lived investors who can voluntarily trade at all times. ${ }^{1}$ Alternative models with finite horizons, on the other hand, build on an OLG framework where investors live for two periods. ${ }^{2}$ While useful to understand some pricing features and the limitations of arbitrage activity, the lifespan of investors is, by construction, fixed in these models. This rigid structure makes difficult to assess how asset prices in economies with different investors' lifespans compare to each other. The present paper contributes to filling this gap by providing a model where investors trade an infinitely lived asset, but have (arbitrary) finite investment horizons, $T$. The model then studies how variations in $T$ affect asset returns in equilibrium, with a special focus on the implications for the informational role of prices, or market efficiency.

The model is based on the dynamic rational expectations equilibrium analysis pioneered by Wang (1994). Competitive investors trade an infinitely-lived asset to maximize utility of lifetime consumption under CARA preferences. There are two types of investors: those who observe private information about the persistent component of the dividend process (informed investors), and those who must infer it from dividends and prices (uninformed investors). Investors also differ in their age. At any point in time, there are $T$ generations of investors coexisting. $T-1$ groups (aged $1,2, \ldots T-1$ ) are still active in the market and can take voluntary positions, while the oldest generation (aged $T$ ) is exiting and must unwind its positions at prevailing prices. The net supply of the asset is random and causes prices to fluctuate for reasons orthogonal to fundamentals. This prevents prices from fully revealing the information observed by informed investors.

Generally speaking, the central finding of the paper is that investment horizons matter a great deal for asset prices, and market efficiency. Along the stable equilibrium of the model, longer horizons increase average prices, reduce price and return volatility, and lowers the risk premium. Moreover, since price volatility is dominated by fundamentals for long horizons, prices are more informative for investors who learn from them. The market is then not only more stable, but also more efficient in the informational sense, in economies with longer investment lifespans. The generalized OLG economy developed here

\footnotetext{
${ }^{1}$ See Grossman and Shiller (1981), and Campbell (2000) for a comprehensive survey.

${ }^{2}$ See De Long et al. (1990); Spiegel (1998).
} 
highlights two key and novel mechanisms that account for these results. The first relates to the "pricing" of risk, which I label the age-adjusted risk aversion effect. As investors live longer, they are willing to absorb the liquidations of the dying generation at lower expected returns, since they can smooth their consumption over more periods and are less exposed to temporary price deviations. The second mechanism relates to the "quantity" of risk that active investors must bear in equilibrium, which I label the risk transfer effect. As horizons increase, the relative size of the dying generation shrinks in relation to active investors (voluntary traders), who then bear less aggregate risk. As both mechanisms work in the same direction, longer horizons unambiguously reduce risk premium, and mitigate volatility arising from supply innovations.

More specifically, the paper makes three contributions. The first is methodological, and corresponds to the characterization of existence, multiplicity, and stability properties of linear equilibria in generalized OLG models. Nesting arbitrary investment horizons and different information structures, the model studies a variety of economies whose equilibrium properties have not been previously addressed. Regarding existence, economies which fail to exhibit linear equilibria for short horizons will always admit equilibria for large enough $T$. As a reverse interpretation, market equilibria can break down as horizons shorten.

Regarding multiplicity, a finite horizon economy generically exhibits two equilibria (whenever equilibria exists), a result consistent with the findings of Spiegel (1998), and Watanabe (2008), for the case where $T=2 .{ }^{3}$ These include a stable, low volatility equilibrium (LVE) where supply innovation have small price impact, and an unstable, high volatility equilibrium (HVE) where they cause large price fluctuations. This paper describes the evolution of pricing moments along these equilibria, as a function of the investment horizon. Along the stable LVE, increases in $T$ lower the price impact of supply and decrease price volatility. As $T \rightarrow \infty$, the LVE converges smoothly to the infinite-horizon economy of Wang (1994). Along the HVE however, longer horizons leads to unbounded increases in price volatility. In the limit, this equilibrium vanishes as $T \rightarrow \infty$. Intuitively, as investors live longer, both the increased willingness to take risks and the smaller proportion of forced liquidations makes the HVE increasingly "difficult" to sustain. While many of these results rely on numerical simulations, I prove analytically novel results for economies with symmetric information. Namely, as $T \rightarrow \infty$, a linear equilibrium always exist, and it is unique.

Second, the paper introduces and analyzes the afore-mentioned mechanisms which are the key drivers of the results. These mechanisms are, to the best of my knowledge, new to the literature. To understand the age-adjusted risk aversion effect, consider the case of infinitely-lived investors. In this economy, the marginal propensity to consume wealth is the ratio between the net and gross rate of interest, $r / R$. This coefficient is precisely how agents price uncertainty about wealth fluctuations -the "age-adjusted" risk aversion parameter corresponds to $\gamma \cdot r / R$, and $\gamma$ is the CARA parameter. ${ }^{4}$ In the other extreme case in which agents live two periods, the marginal propensity to consume wealth is one, and the effective risk aversion equals $\gamma$. In the present model, the pricing of risk depends on the age of the investor. Importantly, as horizons increase, the average age-adjusted risk aversion declines.

The economics behind the risk transfer effect are as follows. Consider once again the infinite-horizon case. Because investors always trade voluntarily, there is no forced transfer of risk between generations,

\footnotetext{
${ }^{3}$ Two equilibria arise in the single asset case, as studied here. In the $N$-risky asset case, there exists $2^{N}$ equilibria.

${ }^{4}$ This is the economy considered by Wang (1994).
} 
and all agents bear the aggregate risk proportionally. In contrast, in a two-period OLG economy, the dying generation (in mass 1/2) must unload all its positions into a single younger generation (also in mass $1 / 2$ ). In other words, the whole aggregate risk must exchange hands every period! The generalized OLG economy studied here essentially spans the whole intermediate region of horizons left out by these cases, showing how increases in $T$ lower the relative transfer of risk from the dying to all other generations.

The third contribution of the paper -and perhaps the most important one-, is the characterization of asset price informativeness and uncertainty as a function of investors' horizons. I study the behavior of asset prices along the stable LVE for three generic economies: a full-information benchmark where all investors are informed about the persistent component; a no-information economy in which all investors learn only from dividends and prices; and the asymmetric-information economy where a relatively small mass of investors has access to private information. A comparison between these economies reveals the following results: a) For long horizons, the asymmetric information economy behaves similarly to the full information benchmark. The low risk environment implied by large $T$ induces active trading by the informed, which impound their knowledge into prices. Uninformed investors extract precise information from prices, which reduces their uncertainty. In this economy, price movements are largely driven by fundamental volatility, and expected returns and return volatility closely mimic the full-information case. b) For short investment horizons, the asymmetric information economy approaches the no-information benchmark. The high risk implied by small $T$ leads informed investors to trade more cautiously, disaggregating information from prices and increasing uncertainty about fundamental asset values for the uninformed. In this economy, price movements are largely driven by supply innovations, and expected returns and return volatility line up closely with the no-information case.

The model presented here is related to the literature on trading in OLG environments. De Long et al. (1990), as well as Spiegel (1998), study economies with 2-period lived investors. Spiegel (1998) is closest to the present paper as in his model all investors are rational, and the random component of returns comes from (rather small) random innovations in the asset supply. Watanabe (2008) extends Spiegel's model to introduce asymmetric information about forthcoming dividends. In all these models however, investor horizons are fixed. Therefore, the discussion on how the economy can transition between episodes of high and low price volatility remains, by construction, relegated to an equilibrium switching argument only. He and Wang (1995), and Cvitanić et al. (2006), study finite horizon economies with incomplete information. Since agents derive utility only from terminal wealth, the age-adjusted risk aversion coincides with the CARA parameter in both papers. Moreover, in these papers all investors grow old simultaneously, so there is no risk transfer from dying to active generations. The two main forces at work in the present paper are therefore quite different.

Other related papers study the impact of short-term investors in the context of 3-period models. In Froot et al. (1992), investors might choose to study information unrelated to fundamentals to the extent it can predict short-term price movements. Kondor (2012) studies an economy with short-term traders, focusing on how public disclosures can simultaneously increase divergence of (rational) beliefs about returns while lowering the conditional uncertainty about fundamentals. Cespa and Vives (2012) focus on how persistent noise trading can generate two equilibria even in a finite horizon economy. Albagli 
(2009) studies the impact of increased fund liquidations during downturns in effectively lowering investors' horizon, and its implications for price informativeness and expected returns. It is of course difficult to compare the results obtained in a fully dynamic model from those derived from finite horizon environments. The key difference with these papers remains in that the present analysis allows for varying investment horizons -indeed, such variation constitutes the baseline of the results discussed- whereas 3-period models have a rigid lifespan structure. ${ }^{5}$

Finally, the work by Chien et al. (2012) is also related. In their model. some investors re-balance portfolios infrequently, leaving the burden of the adjustment to a small group of sophisticated traders. This mechanism amplifies price effects of negative shocks, generating countercyclical risk premium. Their model has symmetric information, infinitely-lived agents and CRRA preferences, so the forces at work are quite different. Nevertheless, a varying mass of investors who absorb risk is a common theme, and so the findings presented here are complementary to their work.

The rest of the paper is organized as follows. Section 2 introduces the model and equilibrium concept. Section 3 presents the characterization of existence, multiplicity, and stability, for symmetric information economies. Section 4 studies the impact of horizons along the stable LVE in the asymmetric information economy, discussing implications for expected returns, price volatility, and uncertainty. Section 5 concludes. All proofs are in the appendix.

\section{A Generalized OLG economy with Asymmetric Information}

\subsection{Basic Setup}

\subsubsection{Securities}

Time is discrete and runs to infinity. There is a risk-free asset in perfectly elastic supply yielding a gross return of $R=1+r$, and one risky asset paying an infinite stream of dividends $\left\{D_{\tau}\right\}_{\tau=1}^{\infty}$. Dividends follows a mean-reverting process with unconditional mean $\bar{F}$ and persistence $\rho_{F}$ (with $0 \leq \rho_{F} \leq 1$ ):

$$
\begin{aligned}
D_{t} & =F_{t}+\varepsilon_{t}^{D}, \text { with } \\
F_{t} & =\left(1-\rho_{F}\right) \bar{F}+\rho_{F} F_{t-1}+\varepsilon_{t}^{F} .
\end{aligned}
$$

$F_{t}$ is the persistent payoff component. Due to disturbances $\varepsilon_{t}^{D}$ and $\varepsilon_{t}^{F}, F_{t}$ is not revealed by dividends.

The risky asset supply is given by $\theta_{t}$, a mean-reverting stochastic process described by

$$
\theta_{t}=\left(1-\rho_{\theta}\right) \bar{\theta}+\rho_{\theta} \theta_{t-1}+\varepsilon_{t}^{\theta}
$$

where $\bar{\theta} \geq 0$ is its unconditional mean, $\rho_{\theta}$ denotes its persistence (with $0 \leq \rho_{\theta} \leq 1$ ), and $\varepsilon_{t}^{\theta}$ is a white noise disturbance. The error vector $\epsilon_{t} \equiv\left[\begin{array}{llll}\varepsilon_{t}^{D} & \varepsilon_{t}^{F} & \varepsilon_{t}^{\theta}\end{array}\right]^{\prime}$ is serially uncorrelated, and follow a joint normal distribution with mean zero, and variance-covariance matrix $\Sigma=\operatorname{diag}\left(\sigma_{D}^{2}, \sigma_{F}^{2}, \sigma_{\theta}^{2}\right)$.

\footnotetext{
${ }^{5}$ In Albagli (2009), changes in horizons are captured through comparative statics in the mass of investors forced to liquidate early due to households' withdrawals.
} 


\subsubsection{Investors}

The mass of investors in the economy is normalized to unity. A fraction $\mu$ of these, labeled uninformed investors, have access to publicly available information only. Letting $\underline{h}_{t} \equiv\left\{h_{t-s}\right\}_{s=0}^{+\infty}$ denote the complete history of variable $h$ up to time $t$, public informationis the history of dividends and prices represented by the filtration $\Omega_{t}^{U}=\left\{\underline{D}_{t}, \underline{P}_{t}\right\}$. The complement share of investors (in mass $1-\mu$ ) are informed: in addition to public information $\Omega_{t}^{U}$, they observe the contemporaneous realization of the persistent component, $F_{t}$.

The population in the economy follows a generalized overlapping generation structure with a stationary age distribution. That is, at time $t$, a mass $1 / T$ of investors aged $T$ is dying, which is replaced by an equal mass of new-born investors who live for $T$ periods. Hence, at any point in time the economy has $T$ different generations of investors coexisting, aged $j=1,2, \ldots T$ years. The mix between informed and uninformed investors is assumed to be the same in each generation, so that the economy displays a constant age/information distribution. Investors maximize utility of lifetime consumption: $\sum_{s=1}^{T} \beta^{s} U\left(C_{t+s}\right)$, where period-utility is given by negative exponential preferences $U(C)=-e^{-\gamma \cdot C}$ with equal CARA coefficient across generations/investor types. All investors are born with exogenous wealth $w_{0}$.

\subsubsection{Asset Markets}

Investors can take long or short positions in the risky asset during active trading years $j=\{1,2 \ldots T-1\}$, for which they can borrow/save unlimited amounts of the risk-free asset. The dying generation aged $T$, however, must liquidate accumulated positions (and consume terminal wealth). Denoting $X_{j, t}^{U}$ and $X_{j, t}^{I}$ the demand of uninformed and informed investors aged $j$ in period $t$, aggregate asset demand is:

$$
A D: X_{t} \equiv \frac{1}{T}\left(\mu \cdot \sum_{j=1}^{T-1} X_{j, t}^{U}+(1-\mu) \cdot \sum_{j=1}^{T-1} X_{j, t}^{I}\right) .
$$

Investors are price-takers and submit price-contingent demand orders (generalized limit orders) to a "Walrasian auctioneer", who then sets a price $P_{t}$ for the risky asset such that all orders are satisfied. Defining the dollar net excess return of investment in the risky asset as $Q_{t+1} \equiv D_{t+1}+P_{t+1}-R P_{t}$, the wealth of investor aged $j$ consuming $C_{j, t}^{i}$ and demanding $X_{j, t}^{i}$ (for $i=\{U, I\}$ ) evolves according to

$$
W_{j+1, t+1}^{i}=\left(W_{j, t}^{i}-C_{j, t}^{i}\right) R+X_{j, t}^{i} Q_{t+1}
$$

\subsection{Equilibrium Characterization}

\subsubsection{Recursive Representation}

The solution approach builds on the standard, 3-step technique used in CARA-normal REE setups. ${ }^{6}$ First, conjecture a linear price function of the underlying state variables. Based on this conjecture, update beliefs (posterior means and variance) of future returns. Second, derive optimal investors' demands. Third, impose market clearing and solve for the conjectured price coefficients in terms of underlying parameters.

\footnotetext{
${ }^{6}$ See Vives (2008) for a textbook discussion.
} 
More formally, for any filtration $\Omega$, let $H(x \mid \Omega): R \rightarrow[0,1]$ denote the conditional posterior cdf of a random variable $x$. Let $(j, i)$ denote the age/information type of each investor in the economy, with $j=\{1,2, \ldots T\}$, and $i=\{U, I\}$, and let the filtration $\Omega_{t}^{U}=\left\{\underline{D}_{t}, \underline{P}_{t}\right\}$ and $\Omega_{t}^{I}=\left\{\underline{D}_{t}, \underline{P}_{t}, F_{t}\right\}$ represent the information available at time $t$ to uninformed and informed investors, respectively. ${ }^{7}$ The equilibrium concept is as follows:

A competitive rational expectations equilibrium is: 1. A price function given by (7), 2. A risky asset demand $X_{j, t}^{i}=x\left(P_{t}, \Omega_{t}^{i}, j\right)$ by investor $(j, i)$, 3. Posterior beliefs $H\left(\Psi_{t} \mid \Omega_{t}^{U}\right)$ and $H\left(\Psi_{t} \mid \Omega_{t}^{I}\right)$ for uninformed and informed investors, respectively, such that $\forall(j, i)$ : (i) Asset demands are optimal given prices and posterior beliefs; (ii) The asset markets clear at all times; and (iii) Posterior beliefs satisfy Bayes law.

To characterize the equilibrium, the evolution of the state variables must be expressed in recursive form. Let $\Psi_{t+1} \equiv\left[\begin{array}{lll}1 & F_{t+1} & \theta_{t+1}\end{array}\right]^{\prime}$, and define $\hat{F}_{t}^{U} \equiv \mathbb{E}\left[F_{t} \mid \Omega_{t}^{U}\right]$ as the uninformed investors' forecast of the persistent component $F_{t}$. Given equations (1), (2), and (3), the evolution of $\Psi_{t+1}$ can be written as

$$
\Psi_{t+1}=A_{\psi} \Psi_{t}+B_{\psi} \epsilon_{t+1}^{U}
$$

where $A_{\psi}$ and $B_{\psi}$ are matrices of proper order, and the vector $\epsilon_{t+1}^{U} \equiv\left[\begin{array}{llll}\varepsilon_{t+1}^{D} & \varepsilon_{t+1}^{F} & \varepsilon_{t+1}^{\theta} & \tilde{F}_{t}^{U}\end{array}\right]^{\prime}$ is the expanded error vector faced by the uninformed investors, who in addition to the exogenous shocks, face uncertainty coming from their own forecast error $F_{t} ; \tilde{F}_{t}^{U} \equiv \hat{F}_{t}^{U}-F_{t}$ (see the Appendix). The evolution of beliefs, optimal demands, and prices, can now be expressed in terms of this recursive representation.

In particular, I conjecture the following linear equilibrium price:

$$
P_{t}=p_{0}+\hat{p}_{F} \hat{F}_{t}^{U}+p_{F} F_{t}+p_{\theta} \theta_{t}
$$

\subsubsection{Investors' Problem}

For an investor aged $j$ in period $t$, with information given by the filtration $\Omega_{t}^{i}$, the problem is given by

$$
\max _{X_{j, t}^{i}, C_{j, t}} \mathbb{E}\left[-\sum_{s=0}^{T-j} \beta^{s} e^{-\gamma C_{j+s, t+s}} \mid \Omega_{t}^{i}\right], \quad \text { s.t. } W_{j+1, t+1}^{i}=\left(W_{j, t}^{i}-C_{j, t}\right) R+X_{j, t}^{i} Q_{t+1}, \quad W_{1, t}^{i}=w_{0} .
$$

This optimization remains analytically tractable as long as the evolution of future wealth, conditional on information, is normally distributed. The value function then takes a known form in terms of its dependence on the first and second conditional moments of investors' beliefs about the state variables driving future returns. With this tractable value function representation, asset demands and consumption/savings policies can be determined in closed form (see the discussion in Wang (1994) for more details).

We now check whether future excess returns, $Q_{t+1}$, are indeed conditionally normal. For informed investors, this is immediate. Because the informed also observe the public information available to the uninformed, they know the value of the current forecast $\hat{F}_{t}^{U}$. Since they also observe $F_{t}$ privately, the price reveals the realization of supply, $\theta_{t}$. It is then straightforward to show that $Q_{t+1}$ is conditionally

\footnotetext{
${ }^{7}$ Whether we allow informed investors to observe the complete history $\underline{F}_{t}$, or just the current value $F_{t}$, is irrelevant since $\left\{\theta_{t}, F_{t}\right\}$ are sufficient statistics for predicting future returns.
} 
gaussian for the informed investors. For the uninformed, beliefs are characterized by a dynamic filter. Note that from the price equation (7), uninformed investors back out a noisy signal about $F_{t}$, after subtracting the constant, as well as the contribution of their own forecasts, to the price. I label this signal the informational content of price, given by $p_{t} \equiv F_{t}+\lambda \cdot \theta_{t}$, with $\lambda \equiv p_{\theta} / p_{F}$. Together with dividends, price signals constitute the public information about the state vector $\Psi_{t}$, and can be written as

$$
S_{t} \equiv\left[\begin{array}{ll}
D_{t} & p_{t}
\end{array}\right]^{\prime}=A_{s} \Psi_{t}+B_{s} \epsilon_{t}^{U}
$$

The next theorem describes the evolution of uninformed investors beliefs, showing that forecast errors follow a normal distribution. Specifically, let $\mathbb{O} \equiv \mathbb{E}\left[\left(\Psi_{t}-\mathbb{E}\left[\Psi_{t} \mid \Omega_{t}^{U}\right]\right)\left(\Psi_{t}-\mathbb{E}\left[\Psi_{t} \mid \Omega_{t}^{U}\right]\right)^{\prime} \mid \Omega_{t}^{U}\right]$ denote the variance of the state vector, conditional on public information. Then,

Theorem 1 (Beliefs with public information): The distribution of the state vector $\Psi_{t}$, conditional on the filtration $\Omega_{t}^{U}=\left\{\underline{D}_{t}, \underline{P}_{t}\right\}$, is normal with mean $\mathbb{E}\left[\Psi_{t} \mid \Omega_{t}^{U}\right]$ and variance $\mathbb{O}$, where

$$
\mathbb{E}\left[\Psi_{t} \mid \Omega_{t}^{U}\right]=A_{\psi} \mathbb{E}\left[\Psi_{t-1} \mid \Omega_{t-1}^{U}\right]+K\left(S_{t}-\mathbb{E}\left[S_{t} \mid \Omega_{t-1}^{U}\right]\right)
$$

and the conditional variance and projection matrix $K$ jointly solve

$$
\begin{aligned}
\mathbb{O} & =\left(I_{3}-K A_{s}\right)\left(A_{\psi} \mathbb{O} A_{\psi}^{\prime}+B_{\psi} \Delta B_{\psi}^{\prime}\right), \\
K & =\left(A_{\psi} \mathbb{O} A_{\psi}^{\prime}+B_{\psi} \Delta B_{\psi}^{\prime}\right) A_{s}^{\prime}\left(A_{s}\left(A_{\psi} \mathbb{O} A_{\psi}^{\prime}+B_{\psi} \Delta B_{\psi}^{\prime}\right) A_{s}^{\prime}+B_{s} \Delta B_{s}^{\prime}\right)^{-1}, \\
\Delta & =\operatorname{diag}\left(\sigma_{D}^{2}, \sigma_{F}^{2}, \sigma_{\phi}^{2}, \mathbb{O}(2,2)\right) .
\end{aligned}
$$

Once we have checked investors' beliefs follow a conditional gaussian distribution, we can state the results characterizing value functions and the optimal consumption and investment policies.

Theorem 2 (consumption/investment policies): Let $W_{j, t}^{I}$ and $W_{j, t}^{U}$ denote wealth of a j-aged informed and uninformed investor, respectively. Let $M_{t} \equiv\left[\begin{array}{lllll}1 & F_{t} & \theta_{t} & \tilde{F}_{t}^{U}\end{array}\right]^{\prime}$ and $M_{t}^{U} \equiv\left[\begin{array}{lll}1 & \hat{F}_{t}^{U} & \hat{\theta}_{t}^{U}\end{array}\right]^{\prime}$ denote the current projection of informed and uninformed investors about the expanded state vector, respectively. Then,

1. The value function and optimal rules of informed investors correspond to

$$
\begin{aligned}
J^{I}\left(W_{j, t}^{I} ; M_{t} ; j ; t\right) & =-\beta^{t} e^{-\alpha_{j} W_{j, t}^{I}-V_{j}^{I}\left(M_{t}\right)}, \\
X_{j, t}^{I} & =\left(\frac{A_{Q}}{\alpha_{j+1} \Gamma_{j+1}^{I}}-\frac{h_{j+1}^{I}}{\alpha_{j+1} \Gamma_{j+1}^{I}}\right) \cdot M_{t}, \\
C_{j, t}^{I} & =c_{j+1}^{I}+\left(\frac{\alpha_{j+1} R}{\alpha_{j+1} R+\gamma}\right) W_{j, t}^{I}+\frac{M_{t}^{\prime} m_{j+1}^{I} M_{t}}{2\left(\alpha_{j+1} R+\gamma\right)} .
\end{aligned}
$$


2. The value function and optimal rules of uninformed investors correspond to

$$
\begin{aligned}
J^{U}\left(W_{j, t}^{U} ; M_{t}^{U} ; j ; t\right) & =-\beta^{t} e^{-\alpha_{j} W_{j, t}^{U}-V_{j}^{U}\left(M_{t}^{U}\right)}, \\
X_{j, t}^{U} & =\left(\frac{A_{Q}^{U}}{\alpha_{j+1} \Gamma_{j+1}^{U}}-\frac{h_{j+1}^{U}}{\alpha_{j+1} \Gamma_{j+1}^{U}}\right) \cdot M_{t}^{U}, \\
C_{j, t}^{U} & =c_{j+1}^{U}+\left(\frac{\alpha_{j+1} R}{\alpha_{j+1} R+\gamma}\right) W_{j, t}^{U}+\frac{M_{t}^{U^{\prime}} m_{j+1}^{U} M_{t}^{U}}{2\left(\alpha_{j+1} R+\gamma\right)},
\end{aligned}
$$

where $c_{j+1}^{I}, c_{j+1}^{U}$, are age/information-type dependent constants.

Future returns, $Q_{t+1}$, depend on the contemporaneous state variables $F_{t}$ and $\theta_{t}$, but also on the uninformed investors' projection about these variables. This can be conveniently reduced to a dependence on the expanded state vector $M_{t} \equiv\left[\begin{array}{lllll}1 & F_{t} & \theta_{t} & \tilde{F}_{t}^{U}\end{array}\right]^{\prime}$, which includes uninformed investors' forecast error about current state variables. ${ }^{8}$ While this vector is perfectly observed by the informed investors, it is observed with noise by the uninformed investors. Conditional on their information however, their forecast error is a zero-mean, normally distributed random variable. Hence, for both investor types, future returns are linear in these projections ( $M_{t}$ for the informed, $M_{t}^{U} \equiv\left[\begin{array}{lll}1 & \hat{F}_{t}^{U} & \hat{\theta}_{t}^{U}\end{array}\right]^{\prime}$ for the uninformed), plus additional white noise error with gaussian distribution. The problem then remains tractable and value functions and optimal policies have the closed-form expression stated above.

Optimal portfolios take the form found in other dynamic CARA-normal models. Consider the informed investors: the term $A_{Q} /\left(\alpha_{j+1} \Gamma_{j+1}^{I}\right)$ is a mean-variance efficient portfolio capturing the tradeoff between expected returns (numerator) and risk (denominator), where $\alpha_{j+1}$ is the age-dependent risk aversion coefficient, and $\Gamma_{j+1}^{I}$ is the renormalized covariance matrix of returns. In simple terms, this ratio is the response of investors' demand to an increase in expected returns. The second term is a hedging component arising from the fact that innovations in returns affect expected returns further into the future. More precisely, the error innovation $\epsilon_{t+1}$ not only affects returns $Q_{t+1}$, but also the value function at $t+1$, giving rise to an additional source of risk (see Wang (1994) for more details). What makes this particular problem different is of course the dependence of these components on the age of the investor.

Barring some special cases commented below, the solution method relies on numerical procedures. Beginning with a known terminal value function for the dying generation, one can iteratively compute the value functions at earlier ages for each investor to find the optimal consumption and investment rules for all the different ages actively interacting in the asset market. Equilibrium prices can then be solved by imposing the market clearing condition:

$$
\frac{1}{T}\left(\mu \cdot \sum_{j=1}^{T-1} X_{j, t}^{U}+(1-\mu) \cdot \sum_{j=1}^{T-1} X_{j, t}^{I}\right)=\theta_{t} .
$$

The price equilibria conjectured in (7) is the solution to a fixed-point problem. Starting from an initial

\footnotetext{
${ }^{8}$ This is because the forecast error of the uninformed about $F_{t}$ is perfectly colinear with her forecast error about $\theta_{t}$.
} 
price vector conjecture $P$, the equilibrium conditions deliver a new price vector $P^{\prime}=F(P)$, where the functional $F(\cdot)$ is implicitly defined by investors' learning and optimization problems of Theorems 1 and 2, together with the market clearing condition (19). An equilibrium is a price vector satisfying:

$$
P^{*}=F\left(P^{*}\right)
$$

In the next two sections I analyze the properties of the equilibrium and discuss the implications of varying investment horizons for particular classes of economies.

\section{Symmetric Information Economies}

This section compares equilibrium characteristics across economies with different investment horizons. I restrict attention here to symmetric information environments, studying the following two benchmark cases: the no-information economy where the mass of uninformed agents is $\mu=1$, and the full-information economy, with $\mu=0$. These cases are more tractable and allow the derivation of some analytical results. Moreover, these economies convey much of the intuition about the mechanisms triggered from variations in horizons, providing a natural starting point for the analysis.

\subsection{Existence and multiplicity of equilibria}

I begin stating results which can be proven analytically. In particular, it is possible to derive existence and multiplicity results for limiting cases of investment horizons.

Proposition 1 (2-period OLG): Let $T=2$,

a) Let $\mu=0$, and define $\sigma_{\theta}^{*} \equiv \frac{\left(R-\rho_{\theta}\right)}{4 \gamma}\left(\sigma_{D}^{2}+\left(\frac{R}{R-\rho_{F}}\right)^{2} \sigma_{F}^{2}\right)^{-1 / 2}$.

a.1) If $\sigma_{\theta}>\sigma_{\theta}^{*}$, linear equilibria does not exist.

a.2) If $\sigma_{\theta} \leq \sigma_{\theta}^{*}$, there are (weakly) two linear equilibria, with price coefficients given by:

$$
\begin{aligned}
& p_{0}=\frac{1}{r}\left[\left(1+p_{F}\right)\left(1-\rho_{F}\right) \bar{F}+p_{\theta}\left(1-\rho_{\theta}\right) \bar{\theta}\right], \quad p_{F}=\frac{\rho_{F}}{R-\rho_{F}}, \\
& p_{\theta}^{+}=-\frac{\left(R-\rho_{\theta}\right) \sigma_{\theta}^{-2}}{4 \gamma}\left(1-\sqrt{1-\left(\frac{\sigma_{\theta}}{\sigma_{\theta}^{*}}\right)^{2}}\right), \\
& p_{\theta}^{-}=-\frac{\left(R-\rho_{\theta}\right) \sigma_{\theta}^{-2}}{4 \gamma}\left(1+\sqrt{1-\left(\frac{\sigma_{\theta}}{\sigma_{\theta}^{*}}\right)^{2}}\right) .
\end{aligned}
$$

b) Let $\mu=1$, and define $\sigma_{\theta}^{* *} \equiv \frac{\left(R-\rho_{\theta}\right)}{4 \gamma}\left(\sigma_{D}^{2}+\left(\frac{R}{R-\rho_{F}}\right)^{2}\left(\sigma_{F}^{2}+\rho_{F}^{2} \sigma_{u}^{2}\right)\right)^{-1 / 2} w^{-1 / 2}$.

b.1) If $\sigma_{\theta}>\sigma_{\theta}^{* *}$, linear equilibria does not exist. 
b.2) If $\sigma_{\theta} \leq \sigma_{\theta}^{* *}$, there are (weakly) two linear equilibria, with price coefficients given by:

$$
\begin{aligned}
& p_{0}=\frac{1}{r}\left[\left(1+\hat{p}_{F}\right)\left(1-\rho_{F}\right) \bar{F}+p_{\theta}\left(1-\rho_{\theta}\right) \bar{\theta}\right], \quad \hat{p}_{F}=\frac{\rho_{F}}{R-\rho_{F}}, \\
& p_{\theta}^{+}=-\frac{\left(R-\rho_{\theta}\right) \sigma_{\theta}^{-2}}{4 \gamma}\left(1-\sqrt{1-\left(\frac{\sigma_{\theta}}{\sigma_{\theta}^{* *}}\right)^{2}}\right), \\
& p_{\theta}^{-}=-\frac{\left(R-\rho_{\theta}\right) \sigma_{\theta}^{-2}}{4 \gamma}\left(1+\sqrt{1-\left(\frac{\sigma_{\theta}}{\sigma_{\theta}^{* *}}\right)^{2}}\right) .
\end{aligned}
$$

where $\sigma_{u}^{2}=\frac{\sigma_{D}^{2}\left(1-\rho_{F}^{2}\right)+\sigma_{F}^{2}}{2 \rho_{F}^{2}}\left(\sqrt{1+\frac{4 \sigma_{D}^{2} \sigma_{F}^{2} \rho_{F}^{2}}{\left(\sigma_{D}^{2}\left(1-\rho_{F}^{2}\right)+\sigma_{F}^{2}\right)^{2}}}-1\right)$, and $w \equiv \frac{\sigma_{D}^{2}+\left(\frac{R}{R-\rho_{F}}\right)^{2}\left(\rho_{F}^{2} \sigma_{u}^{2}+\sigma_{F}^{2}\right)}{\sigma_{D}^{2}+\rho_{F}^{2} \sigma_{u}^{2}+\sigma_{F}^{2}} \geq 1$.

Proposition 1 makes two central points. First, it states that existence of (linear) equilibria is not granted unless we impose parameter restrictions. Indeed, to confront this situation, many authors working in the standard 2-period OLG model have assumed a very small value for the volatility of supply; $\sigma_{\theta}$. The second result is that, whenever equilibria exists, it is generally multiple (two equilibria). These results are in line with the finding of earlier work, ${ }^{9}$ highlighting that the current model nests the standard OLG economy previously studied. I now explain the intuition for each of these results.

The economics behind non-existence are as follows. Imagine we begin conjecturing a small (in absolute magnitude) price coefficient for supply innovations; $p_{\theta}^{\prime}$. When agents live for two periods, their fate is determined in a single trading round. Given the rather high stakes, agents might be unwilling to hold the asset even if they expect future supply shocks to have the modest price impact associated with $p_{\theta}^{\prime}$. To induce investors to absorb supply, a larger price concession might then be needed. But this is consistent with a more negative coefficient $p_{\theta}^{\prime \prime}<p_{\theta}^{\prime}$, which implies even more volatility of future prices. This iteration might go on without bound, depending on parameter values. Only when volatility of supply remains below a threshold ( $\sigma_{\theta}^{*}$ and $\sigma_{\theta}^{* *}$ for the full- and no-information economies), the model admits a linear price conjecture that constitutes an equilibrium. Notice also that whenever $\rho_{F}>0$ (a necessary condition for $F_{t}$ to be predictive about future dividends), the full-information economy allows an equilibrium at a higher critical $\sigma_{\theta}$, since $\sigma_{\theta}^{*}>\sigma_{\theta}^{* *}$ in this case. Intuitively, agents in the full-information economy know more about the asset's fundamental value, and can tolerate more non-fundamental (supply) risk.

Regarding multiplicity, the coefficient $p_{\theta}$ takes two possible values given by the positive and negative roots of the quadratic equation arising form the market-clearing condition. Along the negative root (equations (22) and (24) for the full- and no-information economies), innovations in supply have large, negative price impact. This is the high-volatility equilibrium (HVE). Along the positive root, in contrast, they have a milder effect in the price (equations (21) and (23)), which makes this the low-volatility equilibrium (LVE). Which root obtains in turns determines the value of the constant term $p_{0}$. The coefficient associated with innovations in $F_{t}$, on the other hand, has a unique solution corresponding to the expected present discounted value of dividends. For the full-information economy, these expectations are a function of $F_{t}$, while for the no-information economy they depend on the forecast $\hat{F}_{t}^{U}$.

These equilibria reflect two rational, self-fulfilling outcomes. Imagine investors believe the LVE is

\footnotetext{
${ }^{9}$ See Spiegel (1998), Watanabe (2008), Bacchetta and van Wincoop (2008), and Banerjee (2011).
} 
being played, and will continue to played in the future. Because non-fundamental price fluctuations are relatively modest, investors require low compensation for absorbing supply, which then has minor effects on prices and returns. As a result of this low risk environment, the average price of the security is high, reflected by both a large coefficient $p_{0}$, and by a negative but small value $p_{\theta}$ applied to the average supply $\bar{\theta}$. But the situation might well be the converse. If investors believe the HVE is being played, non-fundamental shocks impose large risks and agents become reluctant to trade. As a result, supply shocks are accommodated through large price concessions, causing the HVE to be self-fulfilling as well. The high risk faced by investors in this equilibrium is compensated through a large average premium (a low, or even negative value of $p_{0}$, and a large negative value $p_{\theta}$ ).

Proposition 2 provides results for the other extreme case in which agents are infinitely lived.

Proposition 2 (infinite horizon limit): Let $\mu=0$, or $\mu=1$. As $T \rightarrow \infty$,

a) A linear equilibrium always exists.

b) The equilibrium is unique (in the linear class).

The results of Proposition 2 are new. Wang (1994), for instance, states that an equilibrium price equation similar to expression (7) can be solved for numerically. Whether this is the case for all possible parameters, or whether the solution is unique, is left an open question.

Since a proof of existence and multiplicity for finite investment horizons $T>2$ is not available, I will proceed hereon mostly discussing results from numerical simulations. Table 1 introduces the baseline parameters that I will use throughout (unless otherwise stated). I have chosen the variance of the persistent dividend process as a normalization (equal to one) and made it relatively persistent $\left(\rho_{F}=0.95\right)$. In comparison, the temporary dividend component is relatively volatile $\left(\sigma_{D}=3\right) .{ }^{10}$ The average net supply of the risky asset, $\bar{\theta}$, is normalized to one, in accordance to the measure of agents in the economy. Its standard deviation $\sigma_{\theta}$ is set to $10 \%$ of its unconditional value.

Table 1: Baseline parameters

\begin{tabular}{cccccccccc}
$\sigma_{D}$ & $\sigma_{F}$ & $\sigma_{\theta}$ & $\rho_{F}$ & $\rho_{\theta}$ & $\gamma$ & $r$ & $\beta$ & $\bar{F}$ & $\bar{\theta}$ \\
\hline 3 & 1 & 0.1 & 0.95 & 0.6 & 1 & 0.05 & $1 /(1.05)$ & 20 & 1
\end{tabular}

Figure 1 plots the first two pricing moments that emerge from the model under these parameters. The top part of the figure shows how these moments depend on investment horizons under the LVE. Panel a) shows the unconditional mean of prices, while panel b) computes price volatility, defined as the standard deviation of future prices $P_{t+1}$, conditional on public information $\left\{\underline{D}_{t}, \underline{P}_{t}\right\}$. The bottom panels plot the behavior of these moments under the HVE. The circled lines correspond to the full-information economy $(\mu=0)$, while the crossed lines denote the no-information economy $(\mu=1)$.

The first thing to note from Figure 1 is that under the baseline parameters, no equilibria exists for an OLG economy with $T=2$ (i.e., $\sigma_{\theta}>\sigma_{\theta}^{*}$ ). The full-information economy exhibits equilibria starting

\footnotetext{
${ }^{10}$ Although the transitory dividend component volatility does not enter the price equation (7) directly, it makes inference of the persistent dividend component $F_{t}$ more difficult for the uninformed investors.
} 
Figure 1: Existence and multiplicity
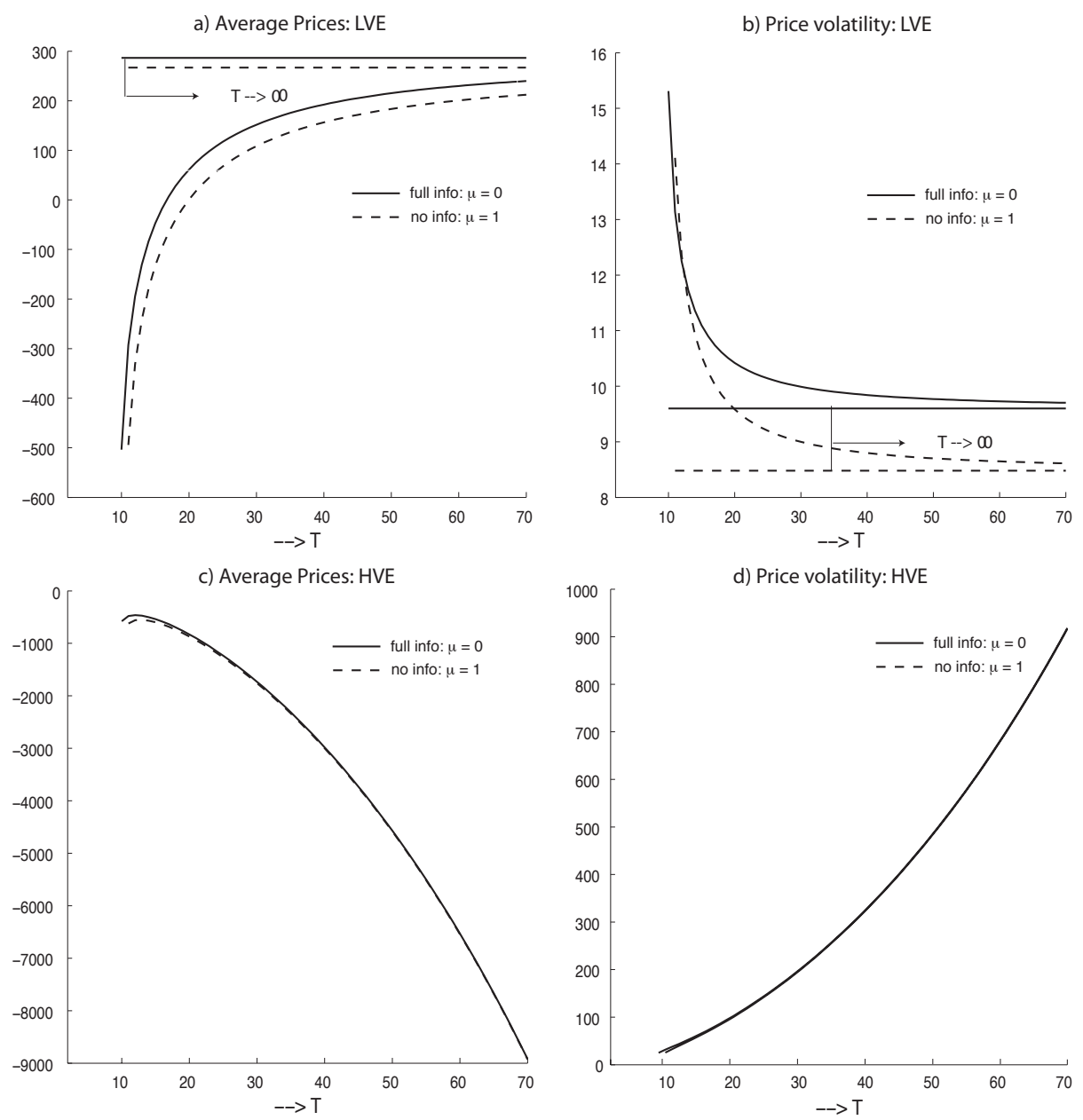

from the critical horizon of $T^{*}=10$ onwards, which coincides with the critical $T^{*}$ for the no-information economy. Regarding multiplicity, the figure shows that for each investment horizon equal or larger than the minimum required for existence, there are 2 equilibria. These numerical results hence confirm that multiplicity is a general feature of dynamic asset markets when agents live for finite periods.

Most importantly, Figure 1 contribute to our understanding of how variations in investor horizons affect the properties of the equilibria in a generalized OLG economy (i.e., $T \in[2, \infty)$ ). Along the LVE, increasing the lifespan of investors increases average prices and reduces price volatility. As mentioned earlier, this is due to two main mechanisms at work: the age-adjusted risk aversion effect, and the risk transfer effect, which I will explain in more detail momentarily. The HVE, in contrast, exhibits the exact opposite features, with average prices falling and volatility increasing with investment horizons. Intuitively, it takes an increasingly volatile security to induce investors to demand the high levels of compensation that are consistent with such volatility (and price discounts) in equilibrium.

In this context, the result of Proposition 2 that multiplicity vanishes as $T \rightarrow \infty$ should be intuitive: a 
second equilibrium with high volatility is a possibility only to the extent that investors are finitely lived, since in this case the threat of a large, adverse price movements late in their lives makes them wary of holding large positions in the asset. ${ }^{11}$ When investors are infinitely lived, however, they can always accommodate a highly volatile asset price by voluntarily buying when it is underpriced, and selling when it is overpriced. Hence, the attractiveness of the highly volatile asset cannot remain a feature of this economy. In equilibrium, only a moderate level of price volatility (and average price discount) is sustainable.

The numerical results of Figure 1 have an important connection with the analytical results of Proposition 2. Namely, while the increase in volatility and drop in average prices are unbounded along the HVE (an equilibrium that vanishes as $T \rightarrow \infty$ ), these moments converge smoothly along the LVE to the values of the infinite horizon economy (straight lines in panels a) and b)). This suggests that the unique equilibrium of the infinite horizon economy corresponds to the limit of the LVE for finite horizons.

Figure 2: Existence regions
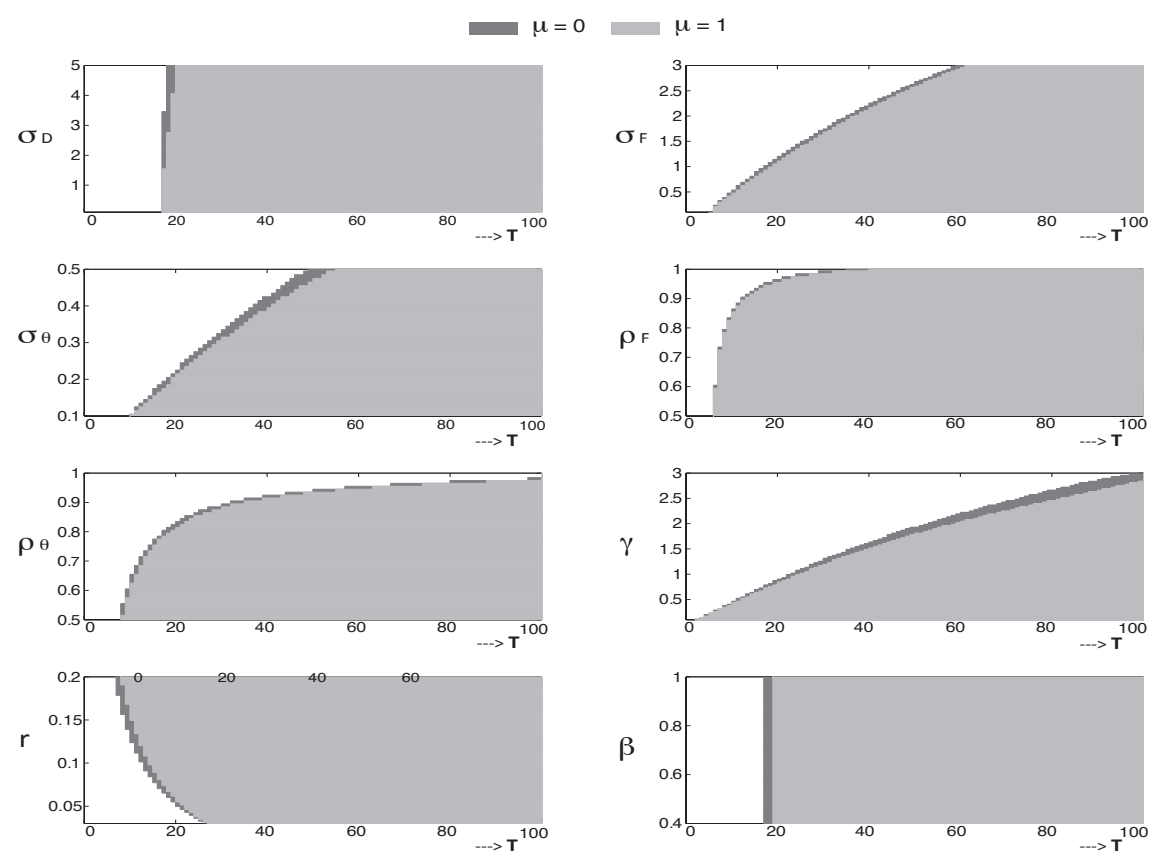

Figure 2 gives a more general picture of the equilibrium existence regions. Starting from the benchmark parameters, each panel shows the dependence of the critical investment horizon $T^{*}$ on a particular parameter, for both the full- and no-information economy. Higher volatility of the dividend process (higher $\sigma_{D}, \sigma_{F}$, or larger persistence $\left.\rho_{F}\right)$ increases the fundamental risk of the security, and require increasing critical horizons $T^{*}$ for existence. Similarly, higher non-fundamental risk related to the random supply shock (larger $\sigma_{\theta}$, or an increase in persistence $\rho_{\theta}$ ) also shift up the minimal horizon. Naturally, risk aversion $\gamma$ increases $T^{*}$, while the converse is true for the interest rate, as prices respond less to funda-

\footnotetext{
${ }^{11}$ Note that Proposition 2 states a sufficient condition for uniqueness, but not a necessary one. In particular, it does not rule out the case that a unique equilibrium might arise with a strictly finite horizon, for $T$ large enough.
} 
mental innovations when investors discount future flows at a higher rate. Finally, although $\beta$ matters for the consumption path chosen by investors, it has no effect in trading decisions (which are wealthindependent), and hence no impact on prices or the critical horizon $T^{*}$. Moreover, for all parameter values, the full-information information economy reaches an equilibrium at a (weakly) lower value of $T^{*}$.

\subsection{Stability}

Another crucial feature that differs across the two possible equilibria is stability. I will define an equilibrium to be stable if, starting from a small perturbation of the price coefficient associated with the supply shock, the resulting price coefficient after one iteration of the agents' optimization leads to a new price vector with a smaller deviation from the initial equilibrium. That is, starting from a small perturbation

$p_{\theta}^{\prime} \neq p_{\theta}^{*}$, an equilibrium is stable if $\left|p_{\theta}^{\prime \prime}-p_{\theta}^{*}\right|<\left|p_{\theta}^{\prime}-p_{\theta}^{*}\right|$, and unstable if $\left|p_{\theta}^{\prime \prime}-p_{\theta}^{*}\right|>\left|p_{\theta}^{\prime}-p_{\theta}^{*}\right|$, where $p_{\theta}^{\prime \prime}$ is the element of the price vector $P^{\prime \prime}$ associated with supply innovations, and $P^{\prime \prime}=F\left(P^{\prime}\right)$.

For the general case, stability can only be inferred from numerical simulations. As discussed in more detail in the appendix, the low-volatility equilibrium is stable according to the above definition, while the high-volatility equilibrium is unstable. A special case which lends easily to an analytical derivation is the symmetric information economy in the standard OLG model with $T=2$.

Proposition 3 (stability): Let $\mu=0$, or $\mu=1$. For $T=2$,

a. The low-volatility equilibrium is stable.

b. The high-volatility equilibrium is unstable.

The result in Proposition 3 sheds light into the fragile nature of the high-volatility equilibrium. Indeed, if the initial price conjecture contains even a slight deviation from the equilibrium value, the "tatonnement" process set in motion by the iteration $P^{\prime \prime}=F\left(P^{\prime}\right)$ will lead the economy further away from the initial equilibrium. In the case where the perturbation is positive (i.e., $p_{\theta}^{\prime}>p_{\theta}^{*}$ ), the economy will converge to the stable LVE. When negative, the economy diverges.

\subsection{Which Equilibrium?}

One of the main appealing properties of the HVE is precisely the capacity to generate high excess volatility, and large correlation among different assets in the case of multiple securities. ${ }^{12}$ As several of the precedent studies indicate, however, there are also some unappealing features about this equilibrium, including the counterintuitive property that the variability of the supply shock $\left(\sigma_{\theta}\right)$ tends to decrease price volatility, while it increases volatility along the LVE. ${ }^{13}$ This remains true in the current model (not reported).

The current study unveils several additional properties that tend to favor the low-volatility as the most suitable candidate for equilibrium selection. First and foremost, volatility along this equilibrium falls as investors' horizons increase, while the opposite is true in the HVE. Not only is it more intuitive that price volatility should decrease with investors' lifespans, but this variation gives the model the potential

\footnotetext{
${ }^{12}$ See the discussion in Spiegel (1998) and Watanabe (2008).

${ }^{13}$ For a comprehensive comparison between different equilibria, see Banerjee (2011).
} 
to generate excess volatility by comparing economies with different investment horizons along the LVE. This finding highlights that, in order to explain periods of high stock market volatility, one need not rely on an equilibrium switching argument -a point I will come back to in section 4 .

Second, while many of the previous studies ignore price levels and focus instead on volatility, it seems like a minimum requirement to have a model that delivers positive prices (at least on average). The present model attains this along the LVE (for long enough horizons) by assuming a positive unconditional mean dividend. However, as panel b) in Figure 1 shows, it is increasingly hard to find positive average prices as horizons increase along the HVE. Third, the present study reveals that while the LVE converges smoothly to the infinite horizon economy, the HVE vanishes as $T \rightarrow \infty$, as shown by Proposition 2. Lastly, the LVE is stable: were the economy to start slightly off-path, successive iterations will restore the LVE. In contrast, the economy always moves away from the HVE even after an arbitrarily small perturbation.

For these reasons, I will focus the attention on the LVE in the remainder of paper.

\subsection{The two key mechanisms}

I now analyze in more detail the two main mechanisms driving the effects of investment horizons on asset prices, in the case of economies with symmetric information.

\subsubsection{Age-adjusted risk aversion effect}

The first mechanism is related to the changes in the pricing of risk induced by changes in $T$, which I will refer to as the age-adjusted risk aversion effect. A key determinant of the risk faced by finitelylived investors in a dynamic trading context are fluctuations in prices. In this respect, the 2-period OLG economy represents a rather extreme case in which investors' fate is determined in a single trading round. When investors live for more periods however, they are less affected by price fluctuations at a particular point in time, since they are not forced to unwind their portfolio at adverse prices -unless they have reached their terminal date. In the words of De long et al. (1990), lengthening horizons is akin to receiving "dividend insurance": by living longer, dividend consumption diminishes the impact non-fundamental risk in the utility of investors.

To understand this mechanism more formally, consider the optimal portfolio decisions of the informed investors in the full-information economy by setting $\mu=0$ (an equivalent argument holds for other information structures). The left panel of Figure 3 plots the age-adjusted risk aversion coefficient, $\alpha_{j+1}$, for an investment horizon $T=30$. Inspection from the numerical results reveal this is the key source of variation in the denominator in the demand expression (14). As shown in the appendix, $\alpha_{j+1}$ can be solved recursively through the equation:

$$
\alpha_{j}=\frac{\gamma \alpha_{j+1} R}{\alpha_{j+1} R+\gamma}
$$

The economics behind this expression is as follows. Fluctuations in future wealth affect the utility of 
investors through its effect on future consumption, given by the marginal propensity to consume wealth:

$$
\frac{\partial C_{j+1, t+1}^{I}}{\partial W_{j+1, t+1}^{I}}=\frac{\gamma \alpha_{j+2} R}{\alpha_{j+2} R+\gamma},
$$

which follows from (15). Using (25), the marginal propensity to consume at age $j+1$ given in (26) must equal $\alpha_{j+1}$, which is precisely the age-adjusted risk aversion used to price return risk $\left(\Gamma_{j+1}^{I}\right)$ in the demand equation (14). For an investor aged $j=29$, this propensity is one, so $\alpha_{j+1}=\gamma \cdot{ }^{14}$ At the other extreme, in an economy with infinitely-lived investors (as in Wang (1994)), $\alpha$ is the stationary solution to (25), corresponding to $\alpha^{*}=\gamma r / R$. More generally, the age-adjusted risk aversion of finitely-lived agents increases with age according to (25), as shown by the left panel of Figure 3.

Figure 3: Age-adjusted risk aversion effect
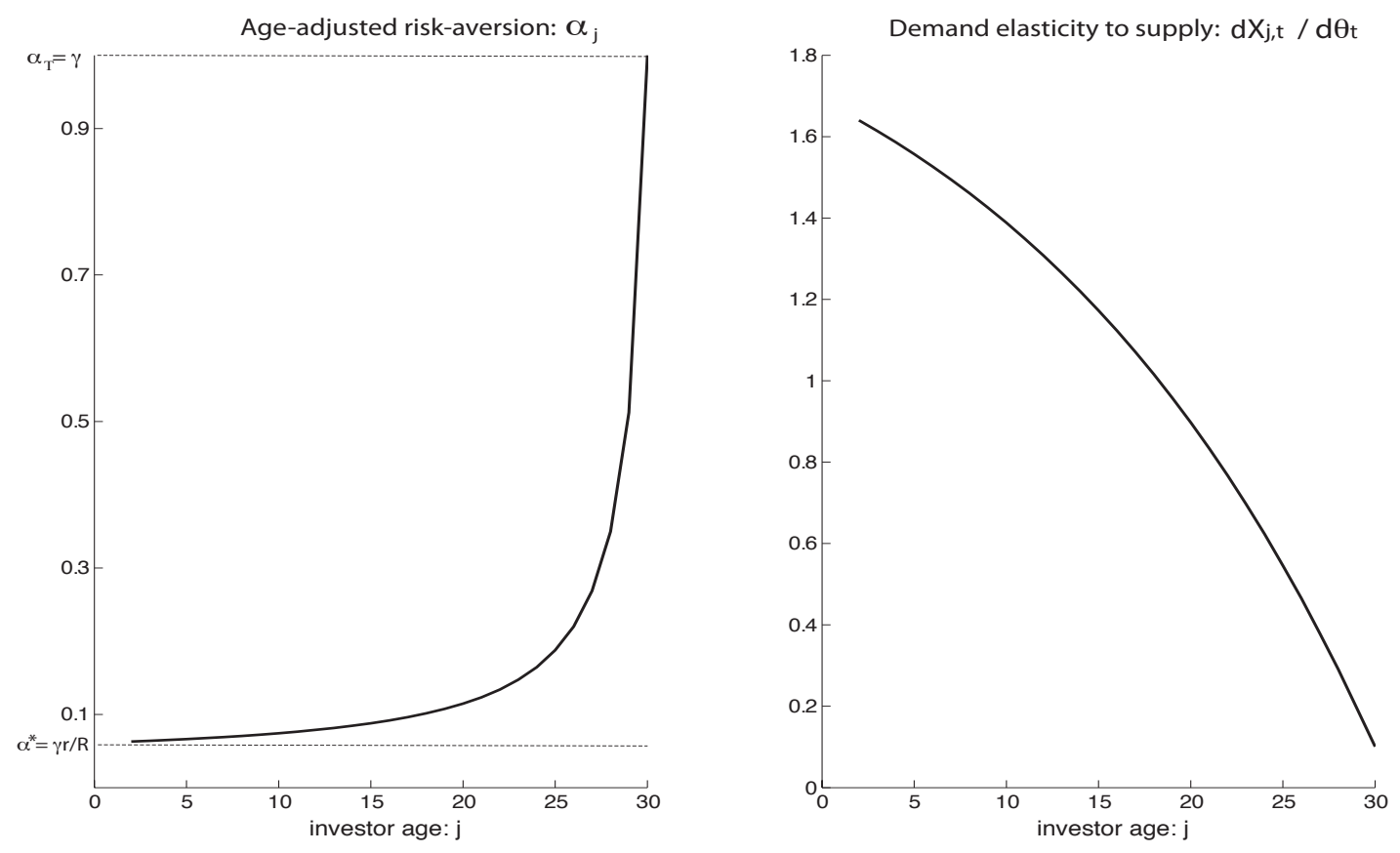

The right panel of Figure 3 shows the elasticity of demands to innovations in supply, as a function of age: as investors get older and risk aversion increases, their relative participation in the market falls. If we now compare economies with different horizons, the average investor in the economy with longer $T$ will be more willing to bear risk, as the average lifespan is higher. To my knowledge, this mechanism has not been studied formally in OLG models of the financial market. A related discussion appears in De long et al. (1990), who state that increasing the age of the 2-period lived investors in their model should lead to more risk-taking and a diminished price impact from "noise traders". This exercise is however not formally carried out in that paper, nor in the successive OLG models which only consider $T=2 .{ }^{15}$

\footnotetext{
${ }^{14}$ Effective risk aversion for this age coincides with the static REE economy with a single consumption period.

${ }^{15}$ Closer in this respect are the dynamic, finite horizon models developed by He and Wang (1995), and Cvitanić et al.
} 


\subsubsection{Risk transfer effect}

The second mechanism is related to changes in the amount of risk that must be absorbed in equilibrium by active generations, induced by changes in $T$. I label this mechanism the risk transfer effect. This mechanism can be illustrated by a convenient decomposition of the market-clearing condition in (19). Continuing with the full-information economy, we can write

$$
\begin{gathered}
\frac{1}{T}\left(X_{1, t}^{I}+X_{2, t}^{I}+\cdots+X_{T-1, t}^{I}\right)= \\
\underbrace{X_{1, t-1}^{I}+X_{2, t-1}^{I}+\cdots+X_{T-1, t-1}^{I}}_{\theta_{t-1}}+\underbrace{\left(1-\rho_{\theta}\right)\left(\bar{\theta}-\theta_{t-1}\right)+\varepsilon_{t}^{\theta}}_{\Delta \theta_{t}} \\
\Rightarrow \quad \frac{1}{T}\left(X_{1, t}^{I}+\Delta X_{2, t}^{I}+\cdots+\Delta X_{T-1, t}^{I}\right)=X_{T-1, t-1}+\Delta \theta_{t}
\end{gathered}
$$

The demand in the left-hand side of (27) is composed of all current active investors. Of these, investors aged $2,3 \cdot \cdot, T-1$ were also present in the previous period, and hence their net demands correspond to $\Delta X_{2, t}^{I}=X_{2, t}^{I}-X_{1, t-1}^{I}$ for the investor currently aged $2, \Delta X_{3, t}^{I}$ for the investor aged 3 , and so on. For all these investors, the change in net positions is voluntary. Only for the investor aged $T-1$ in the previous period the net demand is exogenously set at $-X_{T-1, t-1}^{I}$. In equilibrium, the negative of this amount, plus the supply innovation $\left(1-\rho_{\theta}\right)\left(\bar{\theta}-\theta_{t-1}\right)+\varepsilon_{t}^{\theta}$, must be absorbed by changes in the net positions of all active investors. Hence, there is a risk transfer from a mass of $1 / T$ retirees to a mass of $(T-1) / T$ voluntary investors, or a risk transfer ratio $1 /(T-1)$. For illustrative purposes, the left panel of Figure 4 plots the risk-transfer ratio as a function of the investment horizon.

As the right panel of Figure 3 showed earlier, however, as investors age the increase in (age-adjusted) risk aversion leads to a progressive reduction in asset holdings. The vintage of investors exiting the economy therefore hold less than their "fair share" of the supply. This is made explicit by the right panel of Figure 4, which plots risky asset holdings of the exiting vintage, relative to the average holding. When $T=70$, the exiting vintage have cumulative holdings of about only $7 \%$ of the average investors' holdings. It follows that when this vintage exits the economy, they will increase the supply of the asset by only $(1 / 70) \cdot 7 \% \approx 0.1 \%$. This gradual reduction in the risky positions suggests that the transfer of risk from the dying generation to all others is a smooth process. The fact however remains that, at any point in time, all vintages who hold less than the average share of the supply are transferring risk to those who hold more, an effect induced by the anticipation of each generation that they will in fact die in a finite number of periods. Hence, the risk transfer ratio $1 /(T-1)$ remains as the relevant statistic to account for the importance of this effect.

These two mechanisms (the pricing and quantity of risk) suggest that outcomes in the workhorse OLG economy with $T=2$ might be a bit extreme, and model-specific. On the one hand, current participants in the market must purchase an asset which they must completely unwind in a single future trading round

(2006). As time elapses, agents learn more about the fundamental value of the asset -which is a single terminal payoff. However, since in both models agents derive utility only from consumption of terminal wealth, they price changes in current wealth one-to-one (i.e., the marginal propensity to consume wealth changes is 1). Therefore, the age-adjusted risk aversion coefficient coincides with the CARA parameter. 
Figure 4: Risk transfer effect
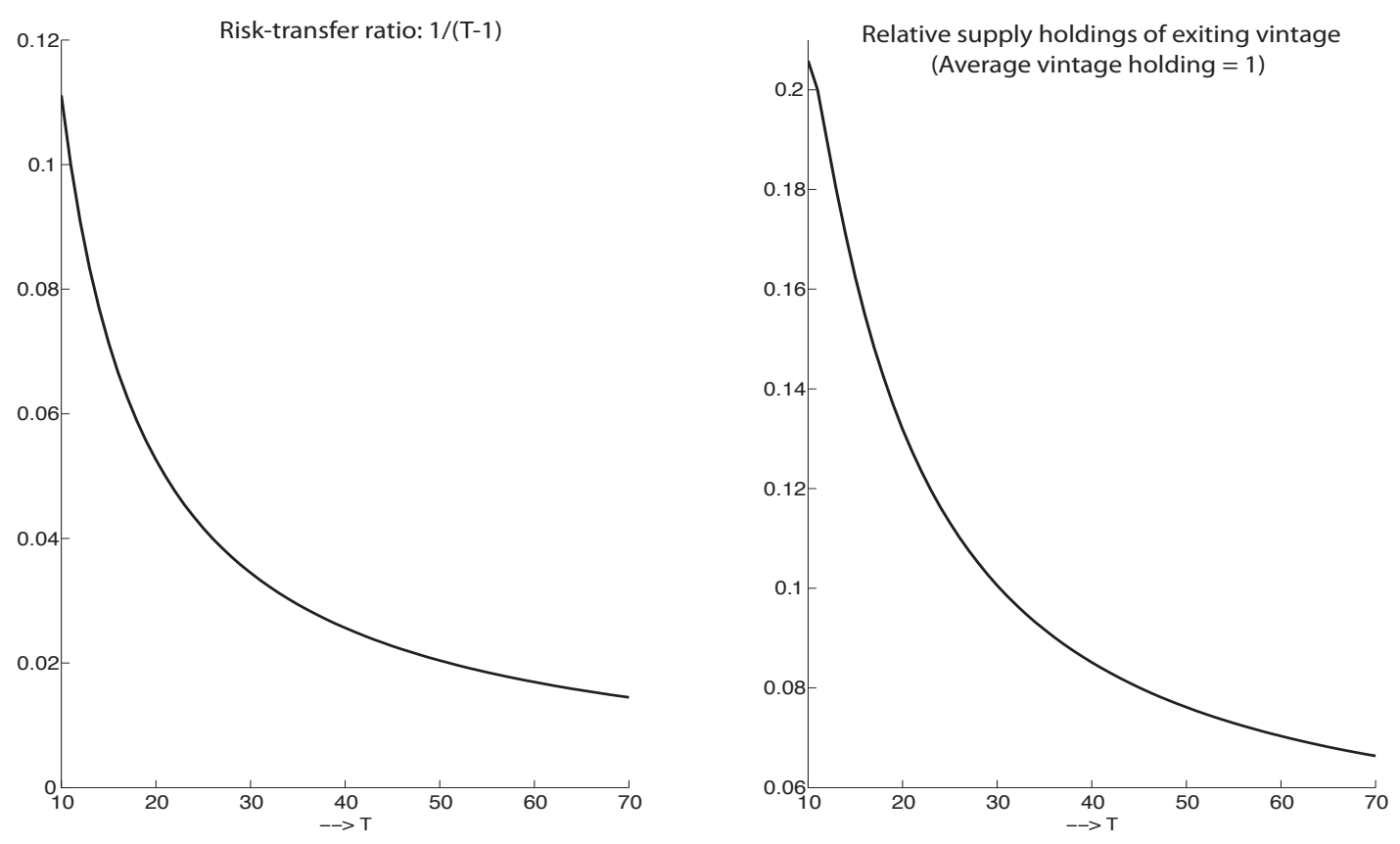

(the age-adjusted risk aversion effect). But moreover, when they do liquidate in the next period, their entire positions must be purchased by a generation with equal mass as them (the risk transfer effect). Simply put, the whole aggregate risk of the economy must forcefully exchange hands every period!

To gauge the relative importance of these two mechanisms, I now perform a decomposition of the change in price volatility through the following exercise. I fix the mass of active (voluntary) investors in the market to one (which corresponds to the infinite horizon economy). I then vary the investment horizon $T$, letting investors' demands change purely as a result of the age-adjusted risk aversion effect. Hence, the exercise captures the behavior of traders that expect to live for finite periods, but eliminates the market impact of their liquidations by artificially normalizing the mass of liquidators to zero -hence, shutting off the risk transfer mechanism.

Figure 5 shows the volatility of prices (conditional on public information) that emerges in these economies. I consider the baseline parameters case, and a case with lower volatility of the random supply, $\sigma_{\theta}=0.05$. As the figure makes clear, both effects are important in delivering the change in price volatility explained by investment horizons. For the baseline parameters (black lines), the contribution of the risk transfer effect is about $33 \%$ of the total price volatility increase produced by shrinking $T$ from 70 to 10 , in the case of the full-information economy. For the no-information economy, the contribution of the risk transfer effect reaches $39 \%$ over the same range. For economies that admit equilibria at lower investment horizons -which is the case when $\sigma_{\theta}=0.05$ (gray lines)- the importance of the risk transfer effect grows larger, since changes in the relative risk transfer ratio, $1 /(T-1)$, become more significant as $T$ approaches the origin. For the full- and no-information economy, in this case the risk transfer effect account for $66 \%$ 
Figure 5: Relative contributions of the effects
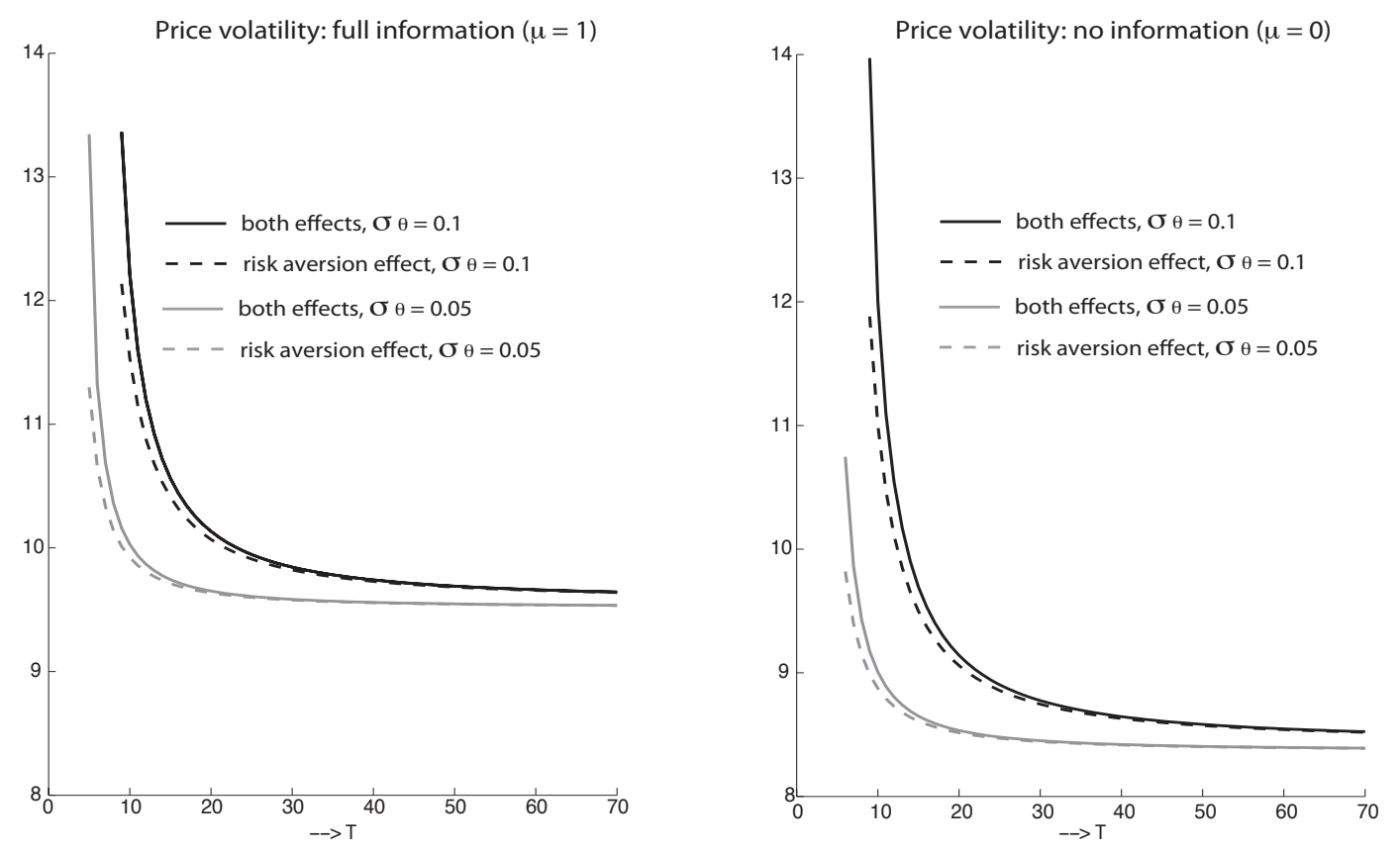

and $42 \%$ of total price volatility changes, respectively.

\section{Asymmetric Information Economies}

This section compares asset pricing moments and the informational role of prices (market efficiency) across economies with different investment horizons, for the case where agents have asymmetric information. The endogenous price signal derived in section $2, p_{t} \equiv F_{t}+\lambda \cdot \theta_{t}$, will be relatively more informative about the persistent dividend component when the ratio $\lambda \equiv p_{\theta} / p_{F}$ is small (in absolute magnitude). But from the discussion in section 3, we learned that as horizons shorten, volatility increases (along the LVE) due to the heightened price response to supply innovations. It follows that in the asymmetric information economy, the informational role of markets will be diminished as the lifespan of investors is reduced. This section focuses on this mechanism, and contrasts the effect of horizons on asset prices with respect to the symmetric information benchmarks analyzed in section 3 .

\subsection{Existence and multiplicity of equilibria}

For comparison purposes, I briefly describe here the results on existence and multiplicity in asymmetric information economies. Although in these economies closed-form solutions for prices -as a function of underlying parameters only- are not available, we can learn about the main equilibrium characteristics from numerical simulations. Qualitatively speaking, the results follow those describe in section 3. Regarding existence, an equilibrium typically does not exist (under the baseline parameters) for very short horizons, 
but emerges as $T$ exceeds a minimum threshold. As a function of the underlying model parameters, the existence regions behave qualitatively similar as those described in Figure 2.

Regarding multiplicity, for any horizon admitting a solution, there are typically two equilibria. While average prices increase and price volatility declines as a function of investment horizons along the LVE, the converse is true along the HVE. Moreover, the LVE is stable to small perturbations, but the HVE is unstable. ${ }^{16}$ For the reasons described in section 3.3, I hereon study the impact of investment horizons on asset prices, and market efficiency, along the LVE.

\subsection{Asset pricing moments}

Figure 6 plots the first two moments of asset prices for three different economies: the full information economy (circled line), the no-information benchmark (crossed line), and the asymmetric information case when $20 \%$ of investors are informed ( $\mu=0.8$, plain line).

The left panel of Figure 6 plots the mean price level. The ordering of mean prices across these economies follow a one-to-one ranking with the amount of fundamental information available to agents: prices are highest for full-information, intermediate for asymmetric information, and lowest for the noinformation economy. Note in particular that for long horizons, average prices in the asymmetric information case approaches the full-information benchmark, but it approaches the no-information economy as horizons shorten.

17

The right panel Figure 6 reveals that price volatility (the standard deviation of future prices, conditional on current public information) might not follow the same ordering. In particular, the no-information economy displays the least amount of price volatility, whereas the asymmetric-information economy is the most volatile. The reason behind this result is intuitive. For the no-information economy, prices convey less information about fundamentals and hence vary less to reflect changes in the underlying persistent dividend component. As the investment horizon shrinks, volatility spikes mostly due to the increased role of supply shocks. In the full-information economy, volatility is larger due to the large impact that fundamentals have on the price when they can be observed without noise. Once again, as horizons grow short, the increased importance of supply shocks raise price volatility.

Just as was the case for mean prices, price volatility in the asymmetric information economy inherits the behavior of the benchmark cases to different degrees, depending on the horizon under consideration. For long horizons, volatility is close to the full information case, although slightly larger since prices respond more to non-fundamental innovations when uncertainty is higher. For relatively short horizons, price volatility gets much closer to the no-information case.

\footnotetext{
${ }^{16}$ For space considerations, I do not report these results explicitly, but they are available upon request.

${ }^{17}$ This result is consistent with Wang (1993), who explicitly compares average prices in an economy with infinitely-lived agents, under different proportions of informed traders. He finds that increasing the fraction of the uninformed strictly decreases the average price level (by reducing the price coefficient $p_{0}$ ). The recent work of Qiu and Wang (2010), and Vayanos and Wang (2012), suggests that average prices can be strictly lower in the asymmetric information economy than in both symmetric information counterparts, as long as investors have background risk exposure that induces trading based on hedging needs. In this sense, it appears that whether the non-informational trading is motivated by background risk, or simply comes from random supply innovations, matters for some asset pricing moments.
} 
Figure 6: Average prices and price volatility
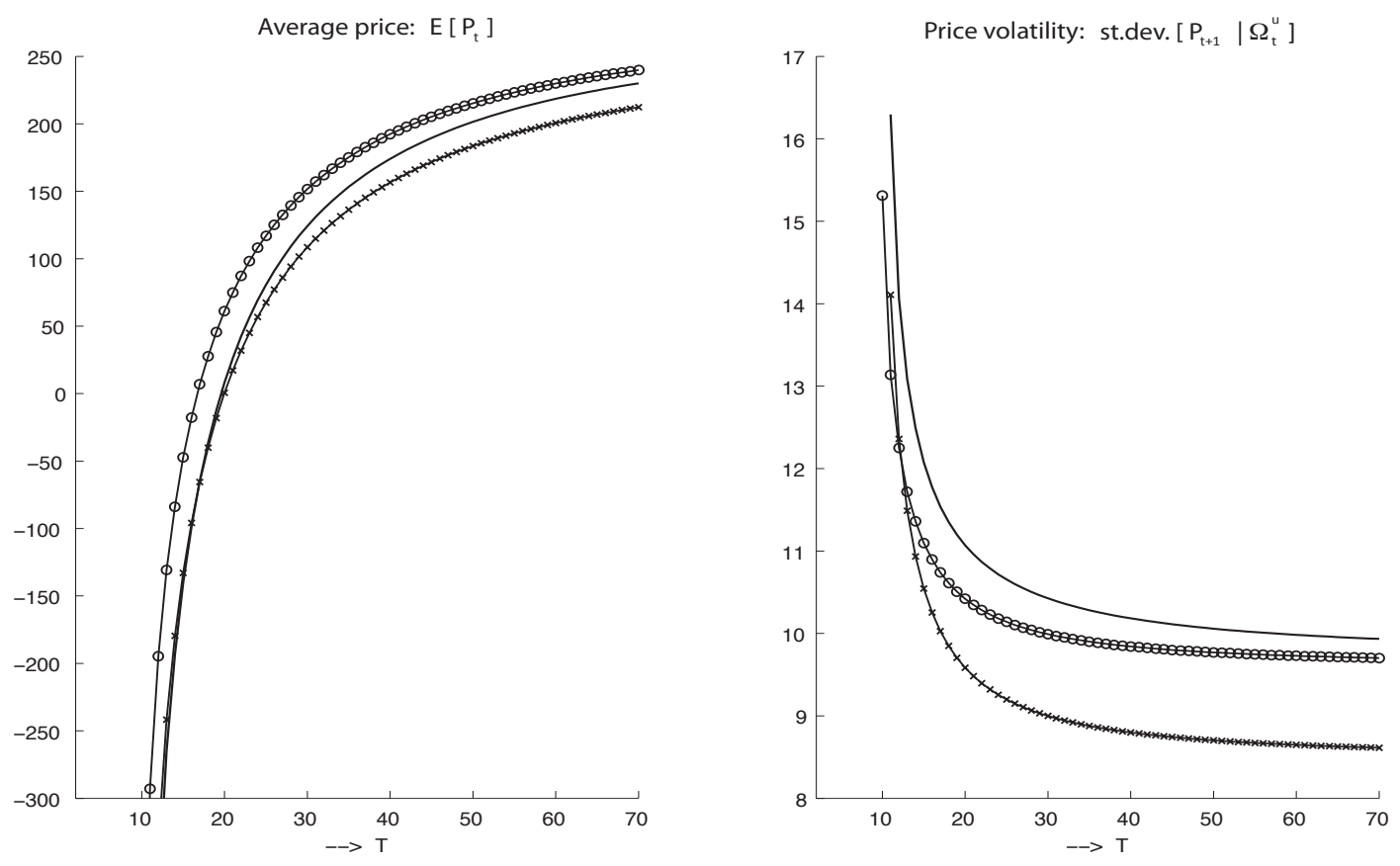

Figure 7: Risk premium and return volatility
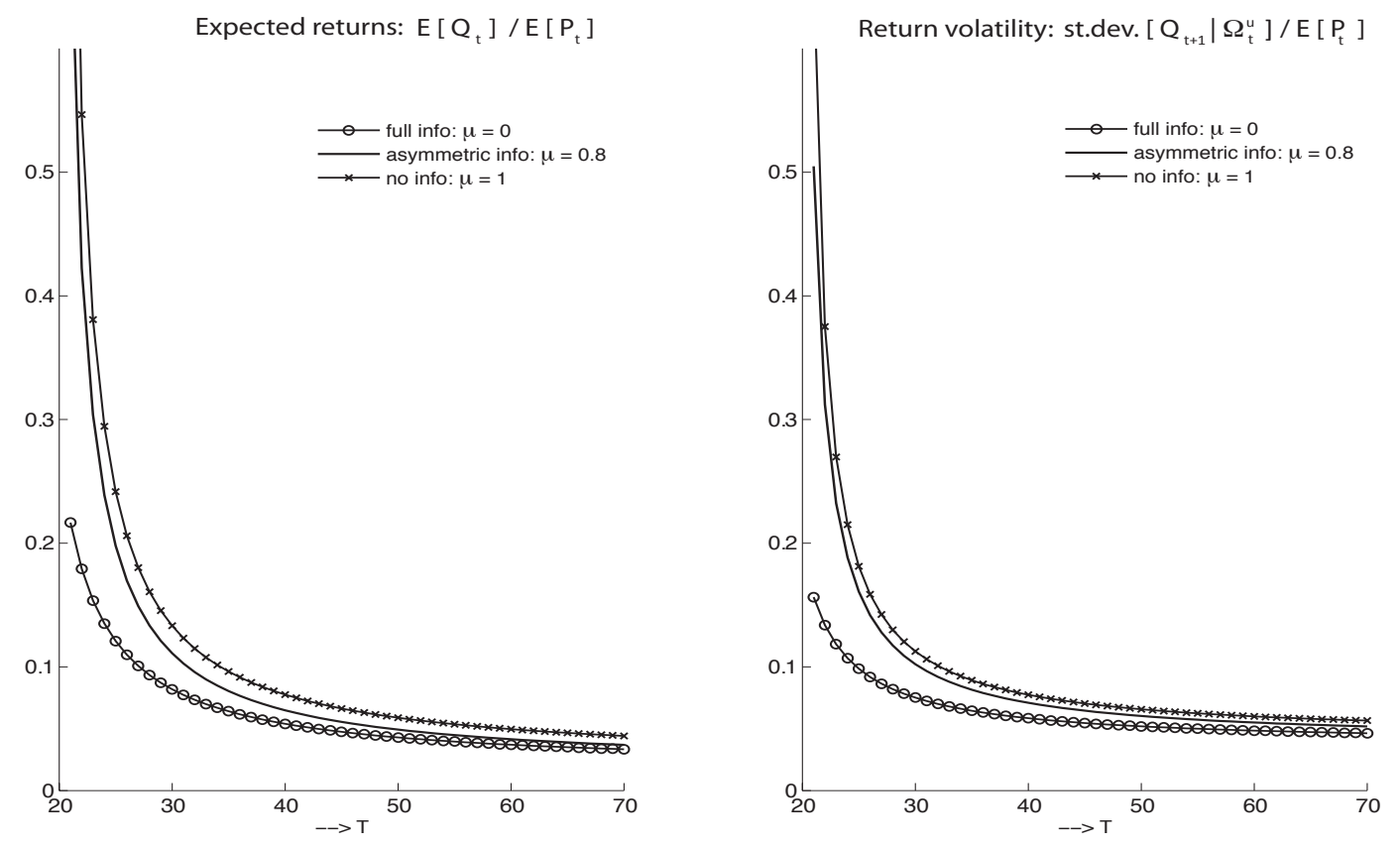
Figure 7 plots the expected return (risk premium) and the volatility of returns across the different economies. Note that the conditional risk premium is time-varying, as it depends on the realization of the state variables. I follow the literature by computing the average risk premium as the ratio between the unconditional dollar return, and the unconditional mean price: $\mathbb{E}\left[Q_{t}\right] / \mathbb{E}\left[P_{t}\right]{ }^{18}$ This definition circumvents the problem that prices can be negative, or arbitrarily close to zero, in linear pricing models. Naturally, for the definition to be meaningful, one needs to restrict attention to economies with strictly positive average prices, which for the baseline parameters occurs for $T \geq 20$. Return volatility is computed as the ratio between the standard deviation of returns, conditional on public information, and the mean price. ${ }^{19}$

The following patterns emerge in this figure which are independent of the parameters chosen. First, both expected returns, and return volatility, are the lowest for the full information economy. Indeed, although prices are relatively volatile in this economy, they are also the highest on average, so the volatility of returns is diminished. Regarding the risk premium, it is intuitive that since investors have the most information about fundamentals in this case, their willingness to participate in the market is the highest, which is reflected by lower required returns. Second, risk premium and return volatility is highest for the no-information economy, since agents face the largest amount of uncertainty regarding the fundamental value of the asset.

Third, and most importantly, the asymmetric information economy lies strictly in between the symmetric information benchmarks. Both expected returns, and return volatility, approach the no-information economy for short horizons. For long horizons, on the other hand, these moments are much closer to the full-information economy. This pattern is to be expected given the discussion of the raw moments of Figure 6. I will now explain in more detail the economic forces behind this last key result.

\subsection{Market efficiency}

Figure 8 plots the decomposition of price volatility (left panel) and the resulting uncertainty of the uninformed investors (right panel), as a function of investment horizons. The share of price fluctuations due to fundamentals is calculated as the fraction of the price variance explained by innovations in $F_{t}$, while the non-fundamental share includes the fraction of price variance coming from both supply shocks, and the forecast errors incurred by the uninformed investors (both shares sum to one).

Clearly, fundamental volatility dominates for long investment horizon, while the converse is true for short lifespans. In consequence, the informational content of prices is diminished as investment horizons shorten, and the uncertainty of the uninformed investors increases. As the right panel of the figure shows, the uncertainty of the uninformed (the standard deviation of $F_{t}$, conditional on the history of public information) nearly doubles as horizons fall from $T=70$, to $T=20$.

Indeed, this figure provides the central intuition for the behavior of investors' required returns, and return volatility, in the asymmetric information economy plotted in Figure 7. In particular, for long investment horizons, the risk premium under asymmetric information comes very close to the returns required in the full-information economy, whereas the no-information economy stands out with a larger

\footnotetext{
${ }^{18}$ This definition is also used in Wang (1993), while Banerjee (2011) discusses his results using mean dollar returns, $\mathbb{E}\left[Q_{t}\right]$.

${ }^{19}$ Return volatility conditional on public information is constant through time, which follows directly from Theorem 1.
} 
Figure 8: Variance decomposition and uncertainty
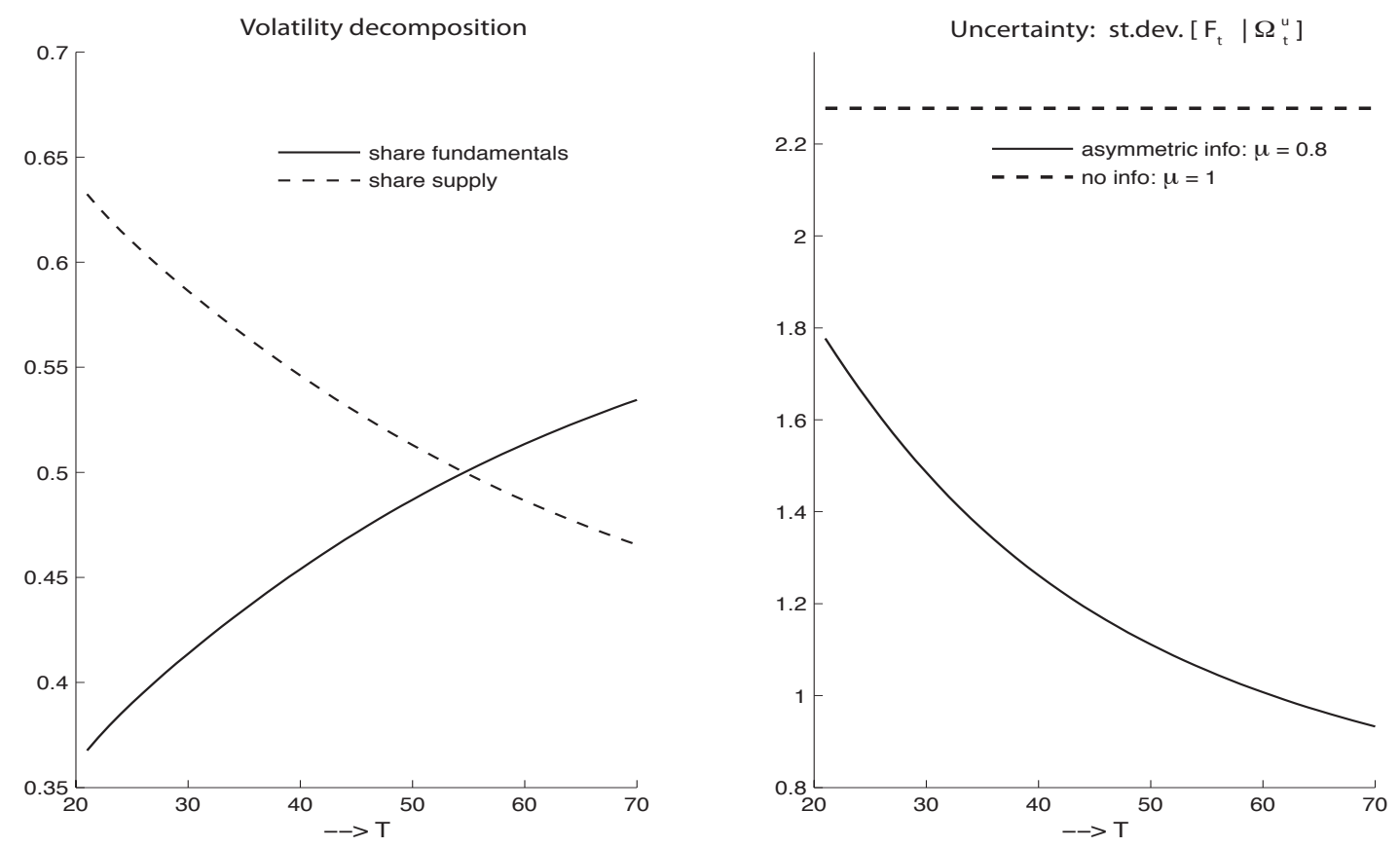

compensation for risk. However, as horizons shorten, the asymmetric information economy approaches the no-information economy, both demanding significantly higher compensation for purchasing the security than the full-information economy.

What explains the apparent switching of the asymmetric information economy between these different benchmark regimes? Precisely the endogenous nature of information in rational expectations environments. In a competitive risk-averse framework, the central force determining how aggressively informed investors react to their private knowledge is risk. For long investment horizons, both the age-adjusted risk aversion, and the risk transfer effect, lead to investor demands which are relatively elastic to expected returns. In consequence, the market is deep and supply innovations have modest price impacts. In response to this low risk environment, competitive CARA-investors who posses superior knowledge will trade on this information aggressively. This implies that the information that is originally observed privately by say, $20 \%$ of the trader population, will mostly find its way into the price. As a direct result, asset prices inherit the behavior exhibited by the full-information, since in this case the average investor is, in effect, pretty well informed in equilibrium.

Conversely, for short investment horizons, the aforementioned effects incite cautious trading and demands become relatively inelastic to private information about future returns. In consequence, information that is private to a group of investors remains private to such group. Shrinking investment horizons hence set in motion a process of information disaggregation from prices, and the knowledge of the population becomes in effect much closer to the no-information benchmark -with asset pricing moments following suit. 
The above facts can also be explained in terms of the excess sensitivity of the risk premium to changes in $T$ in the asymmetric information economy, relative to the symmetric knowledge benchmarks. This excess sensitivity is explained by the interdependence between the uninformed investors' level of uncertainty, and the risk faced by the informed. To understand this link, let's describe in more detail the consequences of a reduction in the demand elasticity of informed investors to private information. If these agents respond less to information, the equilibrium price reveals less information about the fundamental $F_{t}$, and uncertainty about fundamentals is higher for the uninformed. In response, the willingness to make the market is reduced for these agents, which require a larger compensation for bearing a larger amount of risk. In consequence, supply innovations are met with wider price fluctuations. The increased volatility of prices, in turn, increases the risk for the informed, inducing them to react even less strongly to private information. This last observations closes the interdependence, or "spiral", between the risk faced by the informed investors, and uncertainty of the uninformed.

However, one should note that there are limits to the strength of this spiral effect. Indeed, the uncertainty of the uninformed in the asymmetric information economy is capped above by the knowledge of investors in the no-information benchmark, which do learn about the persistent component $F_{t}$ form the observation of dividends. More interestingly, when horizons shrink and the uncertainty of the fundamental process grows larger, at the same time the price is becoming relatively more informative about the noisy supply. Since this second source of price variation takes the center stage for relatively short horizons (Figure 8, left panel), knowing relatively more about this second component becomes increasingly important. In effect, the feedback loop between price volatility and investor uncertainty grows weaker due to this counteracting force. ${ }^{20}$

\subsection{Discussion}

I conclude this section with some remarks on quantitative issues.

\section{Linear pricing limitations:}

First and foremost, CARA-normal models are obviously not designed to capture realistic variations in asset pricing moments, as is the purpose of most of the asset pricing literature using wealth-dependent preferences. This paper is no exception, and the central results should be interpreted as an illustration of the qualitative mechanisms that are triggered by changes in effective investment horizons. That said, the proposed mechanism of varying horizons does seem to play an important role within the confines of the model.

\section{A cyclical interpretation:}

One could, of course, be suspicious about how big the changes in investment horizons need to be for pricing moments to show interesting variations. To asses this question, I will interpret the increased households' withdrawals and fund liquidations that typically occur during crises as an effective shortening

\footnotetext{
${ }^{20}$ For a more detailed description of this counteracting mechanism, see the discussion in Avdis (2011).
} 
of horizons for intermediaries who make the trading decisions.

Under this interpretation, is a reduction from 70 to 20 periods (say, years) a reasonable proxy for what happens at the business cycle frequency? Note that the inverse of the investment horizon corresponds exactly to the fraction of investors which are liquidating forcefully at any given time (the mass of the dying generation). In this light, an increase in liquidations from $1.42 \%$ (i.e., $1 / 70$ ) to $5 \%$ (i.e., 1/20) does not seem exaggerated if one considers the empirical findings of fund liquidations during periods of financial distress. For instance, Ben-David et al. (2012) find that hedge funds reduced their exposure in equity markets in almost 30\% during the 2008:Q3-Q4 contraction, which corresponded roughly to 1\% of all outstanding equities. Importantly, their results indicate that most of this selling was actually forced by investors withdrawing financing. Carhart et al. (2002) study attrition rates in the mutual fund industry, and find that while $3.6 \%$ of funds disappear yearly on average over their sample, the standard deviation is quite high, at 2.4\%. Chen et al. (2008) measure distress selling of troubled -but still alive- mutual funds, and report average distress-driven sales between 0.6-1\% of mutual funds holdings at quarterly frequency (when using the asset-weighted measure of outflows). Moreover, this fraction spikes considerably (nearly doubles) during the mayor episode of financial turbulence covered in their sample: the demise of LTCM in 1998.

Taken together, variations in forced liquidations between $1 \%$ and $5 \%$ per year seem to be in the ball park of the magnitudes reported in these studies for variation at the business cycle frequency.

\section{The importance of endogenous information:}

The last observation relates more generally to the quantitative importance of endogenous information aggregation. As Figure 7 shows, although the asymmetric information economy reacts much more to changes in investment horizons than the full-information economy, one could argue that the no-information economy does a reasonable job in delivering comparable variations in risk premium, and return volatility, as the asymmetric information case. What is then the value added of a (more complex) model that highlights endogenous learning?

The answer is that symmetric information benchmarks have no implications for market efficiency. For the no-information economy, prices contain no additional information about the persistent dividend process - over and above from what can be learned from dividends. In the full-information economy, on the other hand, prices are irrelevant as a source of information since agents already know the value of fundamentals. It is in this respect that the analysis of asymmetric information in financial markets becomes crucial. The results in section 4.3 stress that while return volatility will be high for short horizons, it is the non-fundamental part of volatility that takes the center stage during these episodes. To the extent we believe financial markets matter for the allocation of resources, the loss in informational value of the price system could propagate to the real side of the economy. 


\section{Conclusions}

This paper analyzed the asset pricing implications of finite investment horizons in financial markets. The main message is twofold. First, horizons matter for both the pricing of risk of the average investor (the age-adjusted risk aversion effect) and the amount of risk that must be held in equilibrium by the active investors in a generalized OLG economy (the risk transfer effect). Both mechanism have the potential of delivering interesting variations in expected returns and return volatilities when we compare economies with different length of investors' lifespans.

Second, investment horizons matter for market efficiency. While long horizons incite informed investors to release their private information into prices through the trading process, the high risk environment triggered by short investment horizons can significantly reduce asset price informativeness. In particular, even if the fraction of informed investors is relatively low, the degree of information contained in prices can show ample variations across different horizon regimes. Economies where effective investment horizons are long behave similarly to a hypothetical case in which all investors observe economic fundamentals in real time, while they align much closer with a no-information benchmark when horizons are short. This findings suggests information disaggregation from the price process can be an important mechanism for understanding variations in economic uncertainty more specifically, and market efficiency more generally.

There are several avenues in which one can extend and complement the current analysis. First, although the focus of the paper is studying the asset pricing effects of varying investment horizons, the analysis is limited to the comparison between steady states of different economies, rather than actual time variation in horizons within the same economy. While a very interesting question to study, this type of analysis is difficult to handle within rational expectations models as equilibrium prices lose their linear form. This makes the standard solution methods inapplicable and the analysis becomes intractable.

A related extension which can, in principle, be analyzed under the current framework is the effects of deterministic changes in population demographics. The analysis suggests that a generation of "baby boomers" entering the economy could lead to higher asset prices and reduced uncertainty early in their life, but to declining prices, heightened volatility and poor informational efficiency as the generation approaches old age. Although the equilibrium in this economy would exhibit time-dependent price coefficients, the analysis remains tractable as long as these demographic changes are perfectly anticipated.

Finally, one could also study the incentives to acquire information for different investment horizons. While investors have more incentives to become informed when prices are less reliable, these are also the times when the lifespan over which they plan to use such information is shorter. This leads to non-trivial predictions about the effects of investment horizons on endogenous information acquisition. I leave these important questions for future research. 


\section{References}

[1] Albagli, Elias (2009). "Amplification of Uncertainty in Illiquid Markets," Unpublished Manuscript.

[2] Avdis, Efstathios (2011). "Information Trade-offs in Dynamic Financial Markets," Unpublished Manuscript.

[3] Bacchetta, Philippe, and Eric van Wincoop (2008). "Higher Order Expectations in Asset Pricing," Journal of Money Credit, and Banking, 40, 837-866.

[4] Banerjee, Snehal (2011). "Learning from Prices and the Dispersion in Beliefs," Review of Financial Studies, 24 (9), 3025-68.

[5] Ben-David, Itzhak, Francesco Franzoni and Rabih Moussawi (2012). "Hedge Fund Stock Trading in the Financial Crisis of 20072009," Review of Financial Studies, 25 (1), 1-54.

[6] Campbell, John (2000). "Asset Pricing at the Millennium," Journal of Finance, 55 (4), 1515-1567.

[7] Cvitanić, Jakša, Ali Lazrak, Lionel Martellini, and Fernando Zapatero (2006). "Dynamic Portfolio Choice with Parameter Uncertainty and the Economic Value of Analysts' Recommendations," Review of Financial Studies, 19 (4), 1113-1156.

[8] Carhart, Mark, Jennifer Carpenter, Anthony Lynch, and David Musto (2002). "Mutual Fund Survivorship," Review of Financial Studies, 15 (5), 1439-1463.

[9] Cespa, Giovanni and Xavier Vives (2012). "Expectations, Liquidity and Short-term Trading," Unpublished Manuscript.

[10] Chen, Joseph, Samuel Hanson, Harrison Hong, and Jeremy Stein (2008). "Do Hedge Funds Profit from Mutual-Fund Distress?," Unpublished Manuscript.

[11] Chien, YiLi, Harold Cole and Hanno Lustig (2012). "In the Volatility of the Market Price of Risk due to Intermittent Portfolio Re-balancing?," American Economic Review, 102 (6), 2859-96.

[12] De Long, Bradford, Andrei Shleifer, Lawrence Summers, and Robert Waldman (1990). "Noise Trader Risk in Financial Markets," The Journal of Political Economy, 98 (4), 703-738.

[13] Froot, Kenneth, David Scharfstein, and Jeremy Stein (1992). "Herd on the Street: Informational Inefficiencies in a Market with Short-Term Speculation," Journal of Finance, 47 (4), 1461-84.

[14] Grossman, Sanford, and Robert Shiller (1981). "The Determinants of the Variability of Stock Market Prices," American Economic Review, 71 (2), 222-227.

[15] He, Hua and Jiang Wang (1995). "Differential Information and Dynamic Behavior of Stock Trading Volume," Review of Financial Studies 8 (4), 919-972. 
[16] Kondor, Peter (2012). "The More we Know about Fundamentals, the less we Agree on the Price," Review of Economic Studies, 79 (3), 1175-1207.

[17] Ljungqvist, Lars, and Thomas Sargent (2000). Recursive Macroeconomic Theory. MIT press.

[18] Qiu, Weiyang, and Jiang Wang (2010). "Asset Pricing Under Heterogeneous Information," Unpublished manuscript.

[19] Spiegel, Matthew (1998). "Stock Price Volatility in a Multiple Security Overlapping Generations Model," Review of Financial Studies, 11 (2), 419-447.

[20] Vayanos, Dimitri, and Jiang Wang (2012). "Liquidity and Asset Returns Under Asymmetric Information and Imperfect Competition," Review of Financial Studies, 25 (5), 1339-65.

[21] Vives, Xavier (2008). Information and Leaning in Markets. Princeton University Press.

[22] Wang, Jiang (1993). "A Model of Intertemporal Asset Prices Under Asymmetric Information," Review of Economic Studies, 60 (2), 249-282.

[23] Wang, Jiang (1994). "A Model of Competitive Stock Trading Volume," The Journal of Political Economy, 102 (1), 127-168.

[24] Watanabe, Masahiro (2008). "Price Volatility and Investor Behavior in an Overlapping Generations Model with Information Asymmetry," Journal of Finance, 63 (1), 229-272. 


\section{Appendix}

\section{Proof of Theorems 1 and 2:}

To characterize beliefs, optimal consumption and investment rules, and equilibrium prices, one must first find the matrices $A_{\psi}$ and $B_{\psi}$ in expression (6). From equations (1), (2), and (3), the evolution of $\Psi_{t+1}$ is given by (6) as long as

$$
A_{\psi}=\left[\begin{array}{ccc}
1 & 0 & 0 \\
F_{0} & \rho_{F} & 0 \\
\theta_{0} & 0 & \rho_{\theta}
\end{array}\right], \text { and } B_{\psi}=\left[\begin{array}{cccc}
0 & 0 & 0 & 0 \\
0 & 1 & 0 & 0 \\
0 & 0 & 1 & 0
\end{array}\right]
$$

where $F_{0} \equiv\left(1-\rho_{F}\right) \bar{F}$, and $\theta_{0} \equiv\left(1-\rho_{\theta}\right) \bar{\theta}$. I will prove Theorem's 1 and 2 through the following 4 steps. 1) For given coefficients in the price equation, solve the Bayesian filtering problem of the uninformed, and find the autoregressive process of their forecast errors. 2) Find the recursive representation of the conditional state vectors $M_{t}$ and $M_{t}^{U}$, and the conditional distributions of future excess returns, $Q_{t+1}$. 3) Solve optimal demands, and impose market clearing to find equilibrium prices. The resulting price function is then found by equating the market clearing price to the initial price conjecture.

Step 1: Together with the coefficients in the price equation (7), the recursive representation in (6) leads directly to the results in Theorem 1, which is just the Bayesian updating of beliefs described by the Kalman filter. A derivation of the Kalman filter can be found in most advanced statistics textbooks. ${ }^{21}$ Writing $\mathbb{E}\left[\Psi_{t} \mid \Omega_{t}^{U}\right] \equiv \Psi_{t}^{U}$ and $\Psi_{t}^{U}-\Psi_{t} \equiv \tilde{\Psi}_{t}^{U}$ for notational convenience, the evolution of the uninformed investors' forecast error vector can be found from manipulation of (10):

$$
\begin{aligned}
\tilde{\Psi}_{t+1}^{U} & =A_{U} \cdot \tilde{\Psi}_{t}^{U}+B_{U} \cdot \epsilon_{t+1}^{U}, \quad \text { with } \\
A_{U} & \equiv\left(I_{3}-K A_{s}\right) A_{\Psi}, \text { and } B_{U} \equiv\left(K\left(A_{s} B_{\Psi}+B_{s}\right)-B_{\Psi}\right)
\end{aligned}
$$

But notice that the observation of the price implies forecast errors about $F_{t}$ and $\theta_{t}$ are perfectly colinear for the uninformed; i.e, $F_{t}+\lambda \cdot \theta_{t}=F_{t}^{U}+\lambda \cdot \theta_{t}^{U}$, or $\tilde{\theta}_{t}^{U}=-\lambda^{-1} \cdot \tilde{F}_{t}^{U}$. This allows to rewrite the vector $\tilde{\Psi}_{t+1}^{U}$ as $\tilde{\Psi}_{t+1}^{U}=\left[\begin{array}{lll}0 & \tilde{F}_{t+1}^{U} & \tilde{\theta}_{t+1}^{U}\end{array}\right]^{\prime}=A_{U} \cdot\left[\begin{array}{lll}0 & \tilde{F}_{t}^{U} & \tilde{\theta}_{t}^{U}\end{array}\right]^{\prime}+B_{U} \cdot \epsilon_{t+1}^{U}$, or

$$
\begin{array}{ll}
\tilde{F}_{t+1}^{U}= & \rho_{U} \cdot \tilde{F}_{t}^{U}+b_{U} \cdot \epsilon_{t+1}^{U}, \\
\text { with } & \rho_{U} \equiv A_{U}(2,2)-\lambda^{-1} A_{U}(2,3), \text { and } b_{U} \equiv B_{U}(2,:) .
\end{array}
$$

\footnotetext{
${ }^{21}$ See Ljungqvist and Sargent (2000) for applications in macroeconomics.
} 
Step 2: Using (28), the evolution of the conditional state vectors $M_{t}$ and $M_{t}^{U}$ can now be found:

$$
\begin{aligned}
M_{t+1}= & A_{M} \cdot M_{t}+B_{M} \cdot \epsilon_{t+1}, \\
M_{t+1}^{U}= & A_{M}^{U} \cdot M_{t}^{U}+B_{M}^{U} \cdot \epsilon_{t+1}^{U}, \\
\text { with } & A_{M}=\left[\begin{array}{cccc}
1 & 0 & 0 & 0 \\
\left(1-\rho_{F}\right) \bar{F} & \rho_{F} & 0 & 0 \\
\left(1-\rho_{\theta}\right) \bar{\theta} & 0 & \rho_{\theta} & 0 \\
0 & 0 & 0 & \rho_{U}
\end{array}\right], \quad B_{M}=\left[\begin{array}{ccc}
0 & 0 & 0 \\
0 & 1 & 0 \\
0 & 0 & 1 \\
B_{U}(2,1) & B_{U}(2,2) & B_{U}(2,3)
\end{array}\right], \\
A_{M}^{U} & =A_{\Psi}, \quad B_{M}^{U}=K\left(A_{s} A_{\Psi} l_{0} v_{0}+A_{s} B_{\Psi}+B_{s}\right), \quad l_{0} \equiv\left[\begin{array}{lll}
0 & -1 & -\lambda^{-1}
\end{array}\right]^{\prime}, \text { and } v_{0} \equiv\left[\begin{array}{lll}
0 & 0 & 0
\end{array}\right] .
\end{aligned}
$$

Expressions (29) and (30) can now be used to express the conditional moments of future returns for informed and uninformed investors. Note that the forecast $F_{t}^{U}$ in the price equation (7) can replaced to express prices as a function of current state variables and the forecast error of the uninformed,

$$
P_{t}=p_{0}+p_{1} \cdot F_{t}+p_{2} \cdot \theta_{t}+p_{3} \cdot \tilde{F}_{t}^{U} \equiv P \cdot M_{t}
$$

where $p_{1}=\hat{p}_{F}+p_{F}, p_{2}=p_{\theta}, p_{3}=\hat{p}_{F}$, and $P \equiv\left[\begin{array}{llll}p_{0} & p_{1} & p_{2} & p_{3}\end{array}\right]$. Moreover, writing the dividend in (1) as $D_{t+1}=A_{D} \cdot \Psi_{t+1}+B_{D} \cdot \epsilon_{t+1}$, with $A_{D} \equiv\left[\begin{array}{lll}0 & 1 & 0\end{array}\right], B_{D} \equiv\left[\begin{array}{lll}1 & 0 & 0\end{array}\right]$, the future return $Q_{t+1}$ can now be written as:

$$
\begin{aligned}
Q_{t+1}= & A_{Q} \cdot M_{t}+B_{Q} \cdot \epsilon_{t+1}, \\
\text { with } & A_{Q} \equiv A_{D} A_{M}+P \cdot\left(A_{M}-I_{4} R\right), \text { and } B_{Q} \equiv\left(A_{D}+P\right) \cdot B_{M}+B_{D} .
\end{aligned}
$$

For uninformed investors, the corresponding expression in terms of their conditional expectations and forecast errors is:

$$
\begin{aligned}
& Q_{t+1}=A_{Q}^{U} \cdot M_{t}^{U}+B_{Q}^{U} \cdot \epsilon_{t+1}^{U}, \\
& \text { with } A_{Q}^{U} \equiv A_{Q} m_{0}, \quad B_{Q}^{U} \equiv A_{Q} m_{1} v_{0}+B_{Q} m_{0}^{\prime}, \quad m_{0} \equiv\left[\begin{array}{llll}
1 & 0 & 0 & 0 \\
0 & 1 & 0 & 0 \\
0 & 0 & 1 & 0
\end{array}\right]^{\prime} \text {, and } m_{1} \equiv\left[\begin{array}{llll}
0 & -1 & \lambda^{-1} & 1
\end{array}\right]^{\prime}
\end{aligned}
$$

Expression (32) and (33) confirm that future returns indeed follow a conditional gaussian distribution for both informed and uninformed investors.

Step 3: Optimal investment and consumption policies can now be found by applying a known result on value functions in gaussian-exponential environments (see Vives, 2008). In particular, the conjectured value function at $t+1$ for an informed investor aged $j$ at time $t$ takes the form

$$
J^{I}\left(W_{j+1, t+1}^{I} ; M_{t+1} ; j+1 ; t+1\right)=-\beta^{t+1} \cdot \exp \left\{-\alpha_{j+1} W_{j+1, t}^{I}-\frac{1}{2} M_{t+1}^{\prime} V_{j+1}^{I} M_{t+1}\right\}
$$


The value function at $t$ then takes the form

$$
\begin{aligned}
& J^{I}\left(W_{j, t}^{I} ; M_{t} ; j ; t\right)=\max _{\{X, C\}}-\exp \{-\gamma C\}-\beta \delta_{j+1}^{I} \exp \left\{-\alpha_{j+1} R\left(W_{j, t}^{I}+C\right)-\alpha_{j+1} X A_{Q} M_{t}\right. \\
& \left.\left.-\frac{1}{2} M_{t}^{\prime} v_{j+1}^{I, a a} M_{t}+\frac{1}{2}\left(\alpha_{j+1} X B_{Q}+M_{t}^{\prime} v_{j+1}^{I, a b} M_{t}\right)\left(\Xi_{j+1}^{I}\right)^{-1}\left(\alpha_{j+1} X B_{Q}+M_{t}^{\prime} v_{j+1}^{I, a b} M_{t}\right)^{\prime}\right)\right\}, \text { with } \\
& v_{j+1}^{I, a a} \equiv A_{M}^{\prime} V_{j+1}^{I} A_{M} ; \quad v_{j+1}^{I, b b} \equiv B_{M}^{\prime} V_{j+1}^{I} B_{M} ; v_{j+1}^{I, a b} \equiv A_{M}^{\prime} V_{j+1}^{I} B_{M} ; \\
& \Xi_{j+1}^{I} \equiv \Sigma^{-1}+v_{j+1}^{I, b b}, \text { and } \delta_{j+1}^{I} \equiv\left|\Sigma \cdot \Xi_{j+1}^{I}\right|^{-1 / 2} .
\end{aligned}
$$

The optimal consumption/investment policies can now be found through differentiation. Letting $\Gamma_{j+1}^{I} \equiv$ $B_{Q}\left(\Xi_{j+1}^{I}\right)^{-1} B_{Q}^{\prime}$, we get

$$
\begin{aligned}
X_{j, t}^{I} & =\frac{A_{Q}-h_{j+1}^{I}}{\alpha_{j+1} \Gamma_{j+1}^{I}} \cdot M_{t}, \\
C_{j, t}^{I} & =c_{j+1}^{I}+\left(\frac{\alpha_{j+1} R}{\alpha_{j+1} R+\gamma}\right) \cdot W_{j, t}^{I}+\frac{M_{t}^{\prime} m_{j+1}^{I} M_{t}}{2\left(\alpha_{j+1} R+\gamma\right)}, \text { where } \\
c_{j+1}^{I} & \equiv \log \left(\gamma /\left(\beta \delta_{j+1}^{I} \alpha_{j+1} R\right)\right) /\left(\alpha_{j+1} R+\gamma\right) ; h_{j+1}^{I} \equiv B_{Q}\left(\Xi_{j+1}^{I}\right)^{-1} v_{j+1}^{I, a b^{\prime}}, \text { and } \\
m_{j+1}^{I} & \equiv g_{j+1}^{\prime}\left(\Gamma_{j+1}^{I}\right)^{-1} g_{j+1}+v_{j+1}^{I, a a}-v_{j+1}^{I, a b}\left(\Xi_{j+1}^{I}\right)^{-1} v_{j+1}^{I, a b^{\prime}} .
\end{aligned}
$$

The matrices $V_{j+1}^{I}$ and scalars $\alpha_{j}$, for $j=1,2 \ldots T$, can be solved recursively by setting $\alpha_{T}=\gamma, V_{T}^{I}=0_{4}$, and using the recursive equations found in the f.o.c.s:

$$
\alpha_{j}=\frac{\gamma \alpha_{j+1} R}{\alpha_{j+1} R+\gamma}, \text { and } V_{j}^{I}=m_{t+1}^{I} \cdot\left(\frac{\alpha_{j}}{\alpha_{j+1} R}\right)+2 \cdot i_{1,1} \cdot\left(\gamma c_{j+1}^{I}+\log \frac{\alpha_{j}}{\gamma}\right)
$$

where $i_{1,1}$ is a $4 \times 4$ matrix with all element equal to zero except for the first, which equals one. For the uninformed investors the procedure is similar, but one must replace $M_{t}, A_{Q}, B_{Q}$, and $\Sigma$, for $M_{t}^{U}, A_{Q}^{U}$, $B_{Q}^{U}$, and $\Delta$, respectively. This allows solving investment and consumption policies using the appropriate superscript $U$ instead of $I$.

We can now impose the market clearing condition (19). Note that although the conditional state vector has different dimensions for the informed and uninformed investors, the uninformed forecasts can be replaced by the actual values of the state variables plus the forecast noise. This leads to the following equation that must be satisfied by the price:

$$
\begin{gathered}
P=Y \cdot Z^{-1}, \text { where } \\
Z \equiv\left[\left(A_{M}-I_{4} R\right) \cdot\left(\left(m_{0} \bar{w}_{0}^{U}-m_{1} v_{0} \bar{w}_{1}^{U}\right) \cdot\left(m_{0}^{\prime}+x_{0}\right)+I_{4} \bar{w}_{0}^{I}\right)-B_{M}\left(\left(m_{0}^{\prime} \bar{w}_{1}^{U} \cdot\left(m_{0}^{\prime}+x_{0}\right)+I_{4} \bar{w}_{1}^{I}\right)\right],\right.
\end{gathered}
$$




$$
\begin{aligned}
& Y \equiv\left[A_{\theta}-\left(A_{D} A_{M} m_{0} \bar{w}_{0}^{U}-\left(A_{D} A_{M} m_{1} v_{0}+\left(A_{D} B_{M}+B_{D}\right) m_{0}^{\prime}\right) \bar{w}_{1}^{U}\right) \cdot\left(m_{0}^{\prime}+x_{0}\right)\right. \\
& \left.-A_{D} A_{M} \bar{w}_{0}^{I}+\left(A_{D} B_{M}+B_{D}\right) \bar{w}_{1}^{I}\right], \quad \bar{w}_{0}^{U} \equiv \frac{1}{T} \sum_{s=2}^{T}\left(\alpha_{s} \Gamma_{s}^{U}\right)^{-1}, \quad \bar{w}_{0}^{I} \equiv \frac{1}{T} \sum_{s=2}^{T}\left(\alpha_{s} \Gamma_{s}^{I}\right)^{-1} \text {, } \\
& \bar{w}_{1}^{U} \equiv \frac{1}{T} \sum_{s=2}^{T}\left(\Xi_{s}^{U}\right)^{-1} v_{s}^{U, a b^{\prime}}\left(\alpha_{s} \Gamma_{s}^{U}\right)^{-1}, \bar{w}_{1}^{I} \equiv \frac{1}{T} \sum_{s=2}^{T}\left(\Xi_{s}^{I}\right)^{-1} v_{s}^{I, a b^{\prime}}\left(\alpha_{s} \Gamma_{s}^{I}\right)^{-1}, \\
& A_{\theta} \equiv\left[\begin{array}{llll}
\bar{\theta} & 0 & 1 & 0
\end{array}\right] \text {, and } x_{0} \equiv\left[\begin{array}{lll}
0 & 1 & -\lambda^{-1}
\end{array}\right]^{\prime} \cdot v_{0} \text {. }
\end{aligned}
$$

Equation (40) is the fixed point problem that determines the equilibrium price vector (whenever an equilibrium exists). To see this, notice that both the (1x4) matrix $Y$ and the $(4 \times 4)$ matrix $Z$ depend on an initial conjecture of the price vector $P_{0}$ through their dependence on $A_{M}, B_{M}, A_{Q}, B_{Q}, A_{M}^{U}, B_{M}^{U}$, $A_{Q}^{U}$, and $B_{Q}^{U}$, all function of $P_{0}$. The fixed point in (40) that defines the solution can then be written as $P^{*}=Y\left(P^{*}\right) Z\left(P^{*}\right)^{-1}=F\left(P^{*}\right)$, where the functional $F(\cdot)$ is implicitly defined by the f.o.c's in the dynamic optimization of investors together with the market clearing requirement.

\section{Proof of Proposition 1:}

a) For the full-information economy $(\mu=0)$, the price vector $P^{I}=\left[\begin{array}{lll}p_{0} & p_{F} & p_{\theta}\end{array}\right]$ loads on the state vector $M_{t} \equiv\left[\begin{array}{lll}1 & F_{t} & \theta_{t}\end{array}\right]^{\prime}$. The market clearing condition then reads (dropping age subscripts):

$$
\frac{A_{Q}}{2 \gamma \Gamma^{I}} \Psi_{t}=\left[\begin{array}{lll}
0 & 0 & 1
\end{array}\right] \Psi_{t}
$$

where $A_{Q}=\left[-p_{0} r+p_{F} F_{0}+p_{\theta} \theta_{0}+F_{0} \quad p_{F}\left(\rho_{F}-R\right)+\rho_{F} \quad p_{\theta}\left(\rho_{\theta}-R\right)\right]$, and $\Gamma^{I}=\sigma_{D}^{2}+\left(1+p_{F}\right)^{2} \sigma_{F}^{2}+p_{\theta}^{2} \sigma_{\theta}^{2}$. This yields the following three equations for the price coefficients $p_{0}, p_{F}$ and $p_{\theta}$ :

$$
\begin{aligned}
0 & =-p_{0} r+p_{F} F_{0}+p_{\theta} \theta_{0}+F_{0}, \\
0 & =p_{F}\left(\rho_{F}-R\right)+\rho_{F}, \\
p_{\theta}\left(\rho_{\theta}-R\right) & =2 \gamma\left(\sigma_{D}^{2}+\left(1+p_{F}\right)^{2} \sigma_{F}^{2}+p_{\theta}^{2} \sigma_{\theta}^{2}\right),
\end{aligned}
$$

which yield the quadratic equation for the price coefficient $p_{\theta}$ stated in equations (21) and (22). Clearly, when $\sigma_{\theta}>\sigma_{\theta}^{*}$, the term in the square root is negative and there exist no real solution. Hence, there is no equilibria when $\sigma_{\theta}>\sigma_{\theta}^{*}$, and (weakly) two equilibria whenever $\sigma_{\theta} \leq \sigma_{\theta}^{*}$.

b) For the no-information economy $(\mu=1)$, the price vector $P^{U}=\left[\begin{array}{lll}p_{0} & \hat{p}_{F} & p_{\theta}\end{array}\right]$ loads on the state vector $M_{t}^{U} \equiv\left[\begin{array}{lll}1 & F_{t}^{U} & \theta_{t}\end{array}\right]^{\prime}$ (as the price reveals the random supply $\theta_{t}$ in the absence of informed traders). The market clearing condition in this case reads (dropping age subscripts):

$$
\frac{A_{Q}^{U}}{2 \gamma \Gamma^{U}} M_{t}^{U}=\left[\begin{array}{lll}
0 & 0 & 1
\end{array}\right] M_{t}^{U}
$$

where $A_{Q}^{U}=\left[-p_{0} r+\hat{p}_{F} F_{0}+p_{\theta} \theta_{0}+F_{0} \quad \hat{p}_{F}\left(\rho_{F}-R\right)+\rho_{F} \quad p_{\theta}\left(\rho_{\theta}-R\right)\right]$. To find $\Gamma^{U}=B_{Q}^{U}\left(\Xi_{j+1}^{U}\right)^{-1} B_{Q}^{U \prime}$, note that we can write $B_{Q}^{U}=\left(A_{D}+P^{U}\right) B_{M}^{U}+\tilde{B}_{D}$ in this case, where $B_{M}=K\left(A_{s} B_{\Psi}-A_{s} A_{\Psi} m_{1} v_{0}+B_{s}\right)$ 
(using $\lambda^{-1}=0$ in $m_{1}$ ), and $\tilde{B}_{D}=B_{D}-A_{D} m_{1} j_{0}$, with $j_{0} \equiv\left[B_{U}(2,1) \quad B_{U}(2,2) B_{U}(2,3) \rho_{U}\right]$. The matrix $K$ gives the weighting of the uninformed investors' forecast of the state $A_{\Psi}$ on the vector of signals. When all investors are uninformed, the signal vector includes the dividend -which gives information about the fundamental $F_{t^{-}}$, and the price -which is fully informative about the noisy supply $\theta_{t}$, but contains no information about $F_{t} . K$ can be solved through the iteration presented in equations (11) and (12), which in this case allows to solve for the conditional variance of $F_{t}$ (element $(2,2)$ in the $\mathbb{O}$ matrix) and matrix $K$ in closed form. Labeling $\mathbb{O}(2,2) \equiv \sigma_{u}^{2}$, we can write:

$$
K=\left[\begin{array}{cc}
0 & 0 \\
\frac{\rho_{F}^{2} \sigma_{u}^{2}+\sigma_{F}^{2}}{\sigma_{D}^{2}+\rho_{F}^{2} \sigma_{u}^{2}+\sigma_{F}^{2}} & 0 \\
\theta_{0} & 0
\end{array}\right] \text {, and } \mathbb{O}=\left[\begin{array}{ccc}
0 & 0 & 0 \\
0 & \frac{\sigma_{D}^{2}\left(\rho_{F}^{2} \sigma_{u}^{2}+\sigma_{F}^{2}\right)}{\sigma_{D}^{2}+\rho_{F}^{2} \sigma_{u}^{2}+\sigma_{F}^{2}} & 0 \\
0 & 0 & 0
\end{array}\right]
$$

This gives a quadratic equation for $\sigma_{u}^{2}$, whose positive root corresponds to

$$
\sigma_{u}^{2}=\frac{\sigma_{D}^{2}\left(1-\rho_{F}^{2}\right)+\sigma_{F}^{2}}{2 \rho_{F}^{2}}\left(\sqrt{1+\frac{4 \sigma_{D}^{2} \sigma_{F}^{2} \rho_{F}^{2}}{\left(\sigma_{D}^{2}\left(1-\rho_{F}^{2}\right)+\sigma_{F}^{2}\right)^{2}}}-1\right),
$$

as stated in Proposition 1, part b). We can now write

$$
\Gamma^{U}=\frac{\left(\sigma_{D}^{2}+\left(1+\hat{p}_{F}\right)^{2}\left(\rho_{F}^{2} \sigma_{u}^{2}+\sigma_{F}^{2}\right)\right)^{2}}{\sigma_{D}^{2}+\rho_{F}^{2} \sigma_{u}^{2}+\sigma_{F}^{2}},
$$

which yields the following three equations for the price coefficients $p_{0}, \hat{p}_{F}$ and $p_{\theta}$ :

$$
\begin{aligned}
0 & =-p_{0} r+\left(1+\hat{p}_{F}\right) F_{0}+p_{\theta} \theta_{0}, \\
0 & =\hat{p}_{F}\left(\rho_{F}-R\right)+\rho_{F}, \\
p_{\theta}\left(\rho_{\theta}-R\right) & =2 \gamma\left(w\left(\sigma_{D}^{2}+\left(1+\hat{p}_{F}\right)^{2}\left(\rho_{F}^{2} \sigma_{u}^{2}+\sigma_{F}^{2}\right)\right)+p_{\theta}^{2} \sigma_{\theta}^{2}\right),
\end{aligned}
$$

with $w \equiv \frac{\sigma_{D}^{2}+\left(1+\hat{p}_{F}\right)^{2}\left(\rho_{F}^{2} \sigma_{u}^{2}+\sigma_{F}^{2}\right)}{\sigma_{D}^{2}+\rho_{F}^{2} \sigma_{u}^{2}+\sigma_{F}^{2}} \geq 1$. This yield the quadratic equation for the price coefficient $p_{\theta}$ stated in equations (23) and (24). Clearly, when $\sigma_{\theta}>\sigma_{\theta}^{* *}$, the term in the square root is negative and there exist no real solution. Hence, there is no equilibria when $\sigma_{\theta}>\sigma_{\theta}^{* *}$, and (weakly) two equilibria whenever $\sigma_{\theta} \leq \sigma_{\theta}^{* *}$. This completes the proof.

Note also that since $w \geq 1$, and $\sigma_{u}>0$, we have that $\sigma_{\theta}^{* *} \geq \sigma_{\theta}^{*}$. This means that, as expected, obtaining equilibria in the no-information economy is harder than in the full-information economy, as the range of values for $\sigma_{\theta}$ satisfying the existence condition in the former is a subset of the parameters yielding existence in the latter.

\section{Proof of Proposition 2:}

This proof consists of three steps. 1) Derive the price equation that arises from the market clearing condition. This leads to a quadratic equation for the random supply coefficient $p_{\theta}$ in the price equation (7). I will show here that both roots of the equation depend on a particular coefficient of the value 
function matrix $V$ (the stationary value function matrix in the infinite horizon case). This coefficient is the ninth element of the matrix, associated with the utility (value function) impact of random supply innovations, which I label $V_{\theta}$. 2) Derive a second equation which describes the element $V_{\theta}$ as a function of the price coefficient $p_{\theta}$. An equilibrium is then a pair $\left\{p_{\theta}, V_{\theta}\right\}$ satisfying both equations. 3 ) show that a) an intersection between these functions always exists (part a) of the proposition), and b) it is unique (part b) of the proposition). Since the problem of investors in the no-information economy can always be represented as an equivalent full-information economy with scaled up noise, and the proof is valid for all possible parameter values, I will prove here the proposition for the full-information economy only.

Step 1: In the infinite horizon case with full information $(\mu=0)$, there exists only one type of investor whose asset demand (equation (36)) can be restated (dropping the age and information subscripts) as:

$$
X_{t}=\frac{A_{Q}-B_{Q} \Xi^{-1} v^{a b^{\prime}}}{\alpha \Gamma} \Psi_{t}
$$

where $A_{Q}=\left[-p_{0} r+p_{F} F_{0}+p_{\theta} \theta_{0}+F_{0} \quad p_{F}\left(\rho_{F}-R\right)+\rho_{F} \quad p_{\theta}\left(\rho_{\theta}-R\right)\right]$ and $B_{Q}=\left[\begin{array}{lll}1 & \left(1+p_{F}\right) & p_{\theta}\end{array}\right]$ are the row vectors associated with the loadings of future returns on the vector of contemporary state variables, and future disturbances, respectively. It is straightforward to show that for this economy,

$$
\Xi=\left[\begin{array}{ccc}
\sigma_{D}^{-2} & 0 & 0 \\
0 & \sigma_{F}^{-2} & 0 \\
0 & 0 & \sigma_{\theta}^{-2}+V_{\theta},
\end{array}\right], \quad \Gamma=\sigma_{D}^{2}+\left(1+p_{F}\right)^{2} \sigma_{F}^{2}+p_{\theta}^{2} \frac{1}{\sigma_{\theta}^{-2}+V_{\theta}}, \text { and } \alpha=\frac{\gamma r}{R}
$$

Moreover, from equation (35), it can be shown that the second term in the demand's numerator corresponds to $B_{Q} \Xi^{-1} v^{a b^{\prime}}=\left[\begin{array}{lll}p_{\theta} \frac{V_{1,3}+V_{\theta} \theta_{0}}{\sigma_{\theta}^{-2}+V_{\theta}} & 0 & p_{\theta} \frac{\rho_{\theta} V_{\theta}}{\sigma_{\theta}^{-2}+V_{\theta}}\end{array}\right]$, where $V_{1,3}$ is the 3 rd (and 7 th) term of the (symmetric) matrix $V$. The market clearing condition then reads

$$
\frac{\left[-p_{0} r+p_{F} F_{0}+p_{\theta}\left(\theta_{0} \frac{\sigma_{\theta}^{-2}}{\sigma_{\theta}^{-2}+V_{\theta}}-\frac{V_{1,3}}{\sigma_{\theta}^{-2}+V_{\theta}}\right)+F_{0} \quad p_{F}\left(\rho_{F}-R\right)+\rho_{F} \quad p_{\theta}\left(\rho_{\theta} \frac{\sigma_{\theta}^{-2}}{\sigma_{\theta}^{-2}+V_{\theta}}-R\right)\right.}{\gamma(r / R)\left(\sigma_{D}^{2}+\left(1+p_{F}\right)^{2} \sigma_{F}^{2}+p_{\theta}^{2} \frac{1}{\sigma_{\theta}^{-2}+V_{\theta}}\right)}=\left[\begin{array}{lll}
0 & 0 & 1
\end{array}\right],
$$

which gives rise to three equations determining the price coefficients $p_{0}, p_{F}$ and $p_{\theta}$ :

$$
\begin{aligned}
0 & =-p_{0} r+p_{F} F_{0}+p_{\theta}\left(\theta_{0} \frac{\sigma_{\theta}^{-2}}{\sigma_{\theta}^{-2}+V_{\theta}}-\frac{V_{1,3}}{\sigma_{\theta}^{-2}+V_{\theta}}\right)+F_{0} \\
0 & =p_{F}\left(\rho_{F}-R\right)+\rho_{F} \\
p_{\theta}\left(\rho_{\theta} \frac{\sigma_{\theta}^{-2}}{\sigma_{\theta}^{-2}+V_{\theta}}-R\right) & =p_{\theta}^{2} \frac{\gamma(r / R)}{\sigma_{\theta}^{-2}+V_{\theta}}+\gamma(r / R)\left(\sigma_{D}^{2}+\left(\frac{R}{R-\rho_{F}}\right)^{2} \sigma_{F}^{2}\right) .
\end{aligned}
$$

Expression (43) is the first equation we will use to find the equilibrium pair $\left\{p_{\theta}, V_{\theta}\right\}$. Defining $k \equiv$ 
$(2 \gamma / R)(r / R)^{2}\left(\sigma_{D}^{2}+\left(\frac{R}{R-\rho_{F}}\right)^{2} \sigma_{F}^{2}\right)^{1 / 2}$, and $\sigma^{-2} \equiv \sigma_{\theta}^{-2} \frac{R-\rho_{\theta}}{R}$, we can rewrite the two roots of (43) as

$$
\begin{aligned}
& p_{\theta, 1}^{+}\left(V_{\theta}\right)=-\frac{R^{2}\left(\sigma^{-2}+V_{\theta}\right)}{2 \gamma r}+\frac{R^{2}\left(\sigma^{-2}+V_{\theta}\right)}{2 \gamma r} \sqrt{1-k^{2} \frac{\sigma_{\theta}^{-2}+V_{\theta}}{\left(\sigma^{-2}+V_{\theta}\right)^{2}}} \\
& p_{\theta, 1}^{-}\left(V_{\theta}\right)=-\frac{R^{2}\left(\sigma^{-2}+V_{\theta}\right)}{2 \gamma r}-\frac{R^{2}\left(\sigma^{-2}+V_{\theta}\right)}{2 \gamma r} \sqrt{1-k^{2} \frac{\sigma_{\theta}^{-2}+V_{\theta}}{\left(\sigma^{-2}+V_{\theta}\right)^{2}}} .
\end{aligned}
$$

Step 2: to find the dependence of the value function term $V_{\theta}$ on the model parameters and the price coefficient $p_{\theta}$, we adapt equation (39) to the infinite horizon case to write

$$
V=\frac{m}{R}+2 \cdot i_{1,1} \cdot\left(\gamma c+\log \frac{\alpha}{\gamma}\right)
$$

but since $i_{1,1}$ is zero for all terms besides the first, the expression for the ninth term in $V$ reduces to

$$
\begin{aligned}
V_{\theta} & =\frac{m(3,3)}{R}=p_{\theta}\left(\rho_{\theta} \frac{\sigma_{\theta}^{-2}}{\sigma_{\theta}^{-2}-V_{\theta}}-R\right) \frac{r \gamma}{R^{2}}+V_{\theta} \rho_{\theta}^{2} \frac{\sigma_{\theta}^{-2}}{\sigma_{\theta}^{-2}-V_{\theta}}, \quad \text { or } \\
p_{\theta, 2}\left(V_{\theta}\right) & =-V_{\theta} \frac{R}{\gamma r}\left(1+\frac{\rho_{\theta}\left(1-\rho_{\theta}\right) \sigma_{\theta}^{-2} / R}{\sigma^{-2}+V_{\theta}}\right)
\end{aligned}
$$

Step 3: I now study equations (43) and (46). Figure 9 provides the loci of these equations in the $\left\{V_{\theta}, p_{\theta}\right\}$ space. To find existence, it suffices to show that over the range of values of $V_{\theta}$ for which (43) has a real solution, it intersects either of the two roots of equation (46) at least once. Uniqueness then amounts to showing that this intersection is a singleton. I will prove existence and uniqueness by establishing the following facts:

i) $\partial p_{\theta, 1}^{+}(\cdot) / \partial V_{\theta}>0$;

ii) $\partial p_{\theta, 1}^{-}(\cdot) / \partial V_{\theta}<0, \partial^{2} p_{\theta, 1}^{-}(\cdot) / \partial V_{\theta}^{2}>0$, and $\lim _{V_{\theta} \rightarrow \infty} \partial p_{\theta, 1}^{-}(\cdot) / \partial V_{\theta}=\frac{-R^{2}}{\gamma r}$,

iii) $\partial p_{\theta, 2}(\cdot) / \partial V_{\theta}<0, \partial^{2} p_{\theta, 2}(\cdot) / \partial V_{\theta}^{2}>0$, and $\lim _{V_{\theta} \rightarrow \infty} \partial p_{\theta, 2}(\cdot) / \partial V_{\theta}=\frac{-R}{\gamma r}$, and

iv) At $V_{\theta}^{*},\left|\partial p_{\theta, 1}^{-}\left(V_{\theta}^{*}\right) / \partial V_{\theta}\right|>\left|\partial p_{\theta, 2}\left(V_{\theta}^{*}\right) / \partial V_{\theta}\right|$, where $V_{\theta}^{*}$ satisfies $p_{\theta, 1}^{-}\left(V_{\theta}^{*}\right)=p_{\theta, 2}\left(V_{\theta}^{*}\right)$, whenever an intersection between $p_{\theta, 2}(\cdot)$ and the negative root $p_{\theta, 1}^{-}(\cdot)$ exists.

Facts i)-iii) imply that an intersection between equations (43) and (46) always exist. This is because equation $p_{\theta, 2}(\cdot)$ in $(46)$, which begins in the origin and has a strictly negative slope that converges to a constant, will either intersect the negative root $p_{\theta, 1}^{-}(\cdot)$ in $(45)$ (case 1 ), whose strictly negative slope converges to a constant of larger absolute magnitude than the limit of $p_{\theta, 2}(\cdot)$, or it will intersect the positive root $p_{\theta, 1}^{+}(\cdot)$ in $(44)$ (case 2 ), which has strictly positive slope. 
Figure 9: Infinite horizon economy with symmetric information
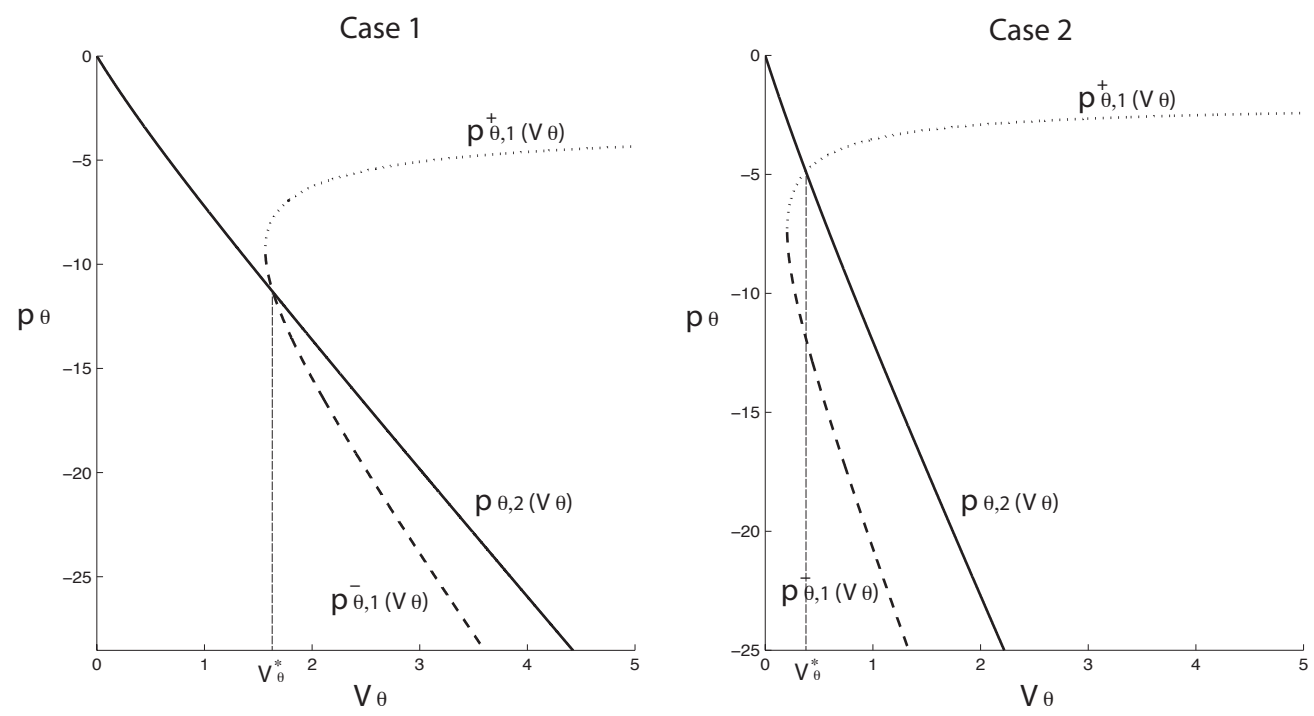

Fact iv) provides the uniqueness result. To see this, notice that in case 1 , the condition that $\left|\partial p_{\theta, 1}^{-}\left(V_{\theta}^{*}\right) / \partial V_{\theta}\right|>\left|\partial p_{\theta, 2}\left(V_{\theta}^{*}\right) / \partial V_{\theta}\right|$ implies that the two lines can only intersect once. If this was not true, then at least in one of these intersections condition iv) would be violated. Regarding case 2 , whenever equation $p_{\theta, 2}(\cdot)$ intersects the positive root $p_{\theta, 1}^{+}(\cdot)$, condition iv) implies it cannot also intersect the negative root $p_{\theta, 1}^{-}(\cdot)$, even once. This is because whenever $p_{\theta, 1}^{+}(\cdot)$ and $p_{\theta, 2}(\cdot)$ intersect, the first intersection with $p_{\theta, 1}^{-}(\cdot)$ would be from above, implying $\left|\partial p_{\theta, 1}^{-}\left(V_{\theta}^{*}\right) / \partial V_{\theta}\right|<\left|\partial p_{\theta, 2}\left(V_{\theta}^{*}\right) / \partial V_{\theta}\right|$, or a violation of condition iv). Hence, condition iv) establishes the global uniqueness of the solution. I now prove each of these claims.

To establish fact i), derive equation (44) w.r.t. $V_{\theta}$, which yields the result immediately. For fact ii), simply derive the negative root $p_{\theta, 1}^{-}(\cdot)$ in $(45)$ twice, which gives

$$
\begin{aligned}
\partial p_{\theta, 1}^{-}(\cdot) / \partial V_{\theta} & =-\frac{R^{2}}{2 \gamma r}\left[1+\frac{2\left(\sigma^{-2}+V_{\theta}\right)-k^{2}}{2\left(\left(\sigma^{-2}+V_{\theta}\right)^{2}-k^{2}\left(\sigma_{\theta}^{-2}+V_{\theta}\right)\right)^{1 / 2}}\right]<0, \\
\partial^{2} p_{\theta, 1}^{-}(\cdot) / \partial V_{\theta}^{2} & =\frac{R^{2}}{2 \gamma r}\left[\frac{k^{2} \sigma_{\theta}^{-2} \rho_{\theta} / R+k^{4} / 4}{\left(\left(\sigma^{-2}+V_{\theta}\right)^{2}-k^{2}\left(\sigma_{\theta}^{-2}+V_{\theta}\right)\right)^{3 / 2}}\right]>0, \quad \text { and } \\
\lim _{V_{\theta} \rightarrow \infty} \partial p_{\theta, 1}^{-}(\cdot) / \partial V_{\theta} & =\frac{-R^{2}}{\gamma r} .
\end{aligned}
$$


Similarly, for fact iii) we derive equation $p_{\theta, 2}(\cdot)$ in $(46)$ twice to find

$$
\begin{aligned}
\partial p_{\theta, 2}(\cdot) / \partial V_{\theta} & =-\frac{R}{\gamma r}\left[1+\frac{\sigma^{-2} \sigma_{\theta}^{-2} \rho_{\theta}\left(1-\rho_{\theta}\right) / R}{\left(\sigma^{-2}+V_{\theta}\right)^{2}}\right]<0, \\
\partial^{2} p_{\theta, 2}(\cdot) / \partial V_{\theta}^{2} & =\frac{R}{\gamma r}\left[\frac{2 \sigma^{-2} \sigma_{\theta}^{-2} \rho_{\theta}\left(1-\rho_{\theta}\right) / R}{\left(\sigma^{-2}+V_{\theta}\right)^{3}}\right]>0, \quad \text { and } \\
\lim _{V_{\theta} \rightarrow \infty} \partial p_{\theta, 2}(\cdot) / \partial V_{\theta} & =\frac{-R}{\gamma r} .
\end{aligned}
$$

Finally, to establish fact iv), let's define the following objects:

$a\left(V_{\theta}\right) \equiv \frac{\sigma_{\theta}^{-2} \rho_{\theta}\left(1-\rho_{\theta}\right) / R}{\left(\sigma^{-2}+V_{\theta}\right)^{2}}, \quad b\left(V_{\theta}\right) \equiv \frac{V_{\theta}}{\sigma^{-2}+V_{\theta}}, c\left(V_{\theta}\right) \equiv 1-k^{2} \frac{\sigma_{\theta}^{-2}+V_{\theta}}{\left(\sigma^{-2}+V_{\theta}\right)^{2}}$, and $d\left(V_{\theta}\right) \equiv \frac{R\left(1-b\left(V_{\theta}\right)\right)}{R-\rho_{\theta} b\left(V_{\theta}\right)}$.

Intersection of equations $p_{\theta, 1}^{-}(\cdot)$ and $p_{\theta, 2}(\cdot)$ at $V_{\theta}=V_{\theta}^{*}$ implies we can write

$$
\begin{gathered}
-\frac{R^{2}}{2 \gamma r}\left(\sigma^{-2}+V_{\theta}^{*}\right)\left(1+c\left(V_{\theta}^{*}\right)^{1 / 2}\right)=-\frac{R}{\gamma r} V_{\theta}^{*}\left(1+a\left(V_{\theta}^{*}\right)\right), \quad \text { or } \\
c\left(V_{\theta}^{*}\right)=\frac{4}{R} b\left(V_{\theta}^{*}\right)\left(1+a\left(V_{\theta}^{*}\right)\right)\left(\frac{b\left(V_{\theta}^{*}\right)\left(1+a\left(V_{\theta}^{*}\right)\right)}{R}-1\right)+1 .
\end{gathered}
$$

We now compare the slopes between equations $p_{\theta, 1}^{-}(\cdot)$ and $p_{\theta, 2}(\cdot)$ at $V_{\theta}=V_{\theta}^{*}$. Manipulation of $\partial p_{\theta, 1}^{-}\left(V_{\theta}^{*}\right) / \partial V_{\theta}$ using the equality condition in (48) allows to write (omitting the dependence on $V_{\theta}$ )

$$
\partial p_{\theta, 1}^{-}(\cdot) /\left.\partial V_{\theta}\right|_{V_{\theta}=V_{\theta}^{*}}=-\frac{1}{\gamma r} \frac{\left[b^{2}(1+a)^{2}+b(1+a) \frac{R-b(1+a)}{R} \rho_{\theta}\right]}{\frac{2}{R} b(1+a)-1},
$$

while the slope of equation $p_{\theta, 2}(\cdot)$ takes the form

$$
\partial p_{\theta, 2}(\cdot) /\left.\partial V_{\theta}\right|_{V_{\theta}=V_{\theta}^{*}}=-\frac{R}{\gamma r}(1+a(1-b)) .
$$

Proving condition iv) then amounts to establishing

$$
\frac{\left[b^{2}(1+a)^{2}+b(1+a) \frac{R-b(1+a)}{R} \rho_{\theta} h\right]}{\frac{2}{R} b(1+a)-1}>1+a(1-b) .
$$

but notice that $a(\cdot)$ can be expressed as $\rho_{\theta}(1-b(\cdot)) \frac{1-\rho_{\theta}}{R-\rho_{\theta}}$. Using this last transformation in (49) and (50) gives after some (tedious) manipulation, the result in (51). This completes the proof.

\section{Proof of Proposition 3:}

Since the problem of investors in the no-information economy can always be represented as an equivalent full-information economy with scaled up noise, I will prove the proposition for the full-information economy 
only. Let $P^{*} \equiv\left[\begin{array}{lll}p_{0}^{*} & p_{F}^{*} & p_{\theta}^{*}\end{array}\right]$ be an equilibrium price vector, and $P^{\prime} \equiv\left[\begin{array}{lll}p_{0}^{*} & p_{F}^{*} & p_{\theta}^{\prime}\end{array}\right]$ represent a perturbation from the equilibrium. When $T=2$, the value function matrix $V$ is the zero matrix, and $\alpha=1$. Let $m \equiv 2 \frac{\gamma}{R-\rho_{\theta}}\left(\sigma_{D}^{2}+\left(\frac{R}{R-\rho_{F}}\right)^{2} \sigma_{F}^{2}\right)^{1 / 2}$. The solution for the price coefficient associated with the supply shock, $p_{\theta}$, is then given by the following roots

$$
\begin{aligned}
& p_{\theta}^{*,+}=-\frac{\sigma_{\theta}^{-2}\left(R-\rho_{\theta}\right)}{2 \gamma}\left(1-\sqrt{1-m^{2} \sigma_{\theta}^{2}}\right), \\
& p_{\theta}^{*,-}=-\frac{\sigma_{\theta}^{-2}\left(R-\rho_{\theta}\right)}{2 \gamma}\left(1+\sqrt{1-m^{2} \sigma_{\theta}^{2}}\right) .
\end{aligned}
$$

Let's now analyze the effects of perturbing the equilibrium by setting $p_{\theta}^{\prime} \neq p_{\theta}^{*}$. From the agents optimization and market clearing condition, we get a new implied price coefficient $p_{\theta}^{\prime \prime}$ given by

$$
p_{\theta}^{\prime \prime}=-\frac{\gamma\left(\sigma_{D}^{2}+\left(\frac{R}{R-\rho_{F}}\right)^{2} \sigma_{F}^{2}\right)}{R-\rho_{\theta}}-\frac{\left(p_{\theta}^{\prime}\right)^{2}}{R-\rho_{\theta}},
$$

from which we can subtract the initial perturbation $p_{\theta}^{\prime}$ to write

$$
p_{\theta}^{\prime \prime}-p_{\theta}^{\prime}=-\left(p_{\theta}^{\prime}-p_{\theta}^{*}\right)\left(1+\frac{\gamma \sigma_{\theta}^{2}\left(p_{\theta}^{\prime}+p_{\theta}^{*}\right)}{R-\rho_{\theta}}\right) .
$$

If we now evaluate this expression at $p_{\theta}^{\prime} \rightarrow p_{\theta}^{*}$ along the low volatility equilibrium in the positive root (equation (52)), we find

$$
p_{\theta}^{\prime \prime}-\left.p_{\theta}^{\prime}\right|_{p_{\theta}^{\prime} \rightarrow p_{\theta}^{*,+}}=-\left(p_{\theta}^{\prime}-p_{\theta}^{*}\right) \sqrt{1-m^{2} \sigma_{\theta}^{2}},
$$

and so $\left|p_{\theta}^{\prime \prime}-p_{\theta}^{*}\right|<\left|p_{\theta}^{\prime}-p_{\theta}^{*}\right|$ and the equilibrium is stable. Along the high-volatility equilibrium in the negative root (equation (53)), we get

$$
p_{\theta}^{\prime \prime}-\left.p_{\theta}^{\prime}\right|_{p_{\theta}^{\prime} \rightarrow p_{\theta}^{*,-}}=\left(p_{\theta}^{\prime}-p_{\theta}^{*}\right) \sqrt{1-m^{2} \sigma_{\theta}^{2}}
$$

and so $\left|p_{\theta}^{\prime \prime}-p_{\theta}^{*}\right|>\left|p_{\theta}^{\prime}-p_{\theta}^{*}\right|$ and the equilibrium is unstable. This completes the proof.

\section{Numerical solution method:}

I now describe the numerical methodology used to find the equilibrium coefficients in the price function of equation (7). The problem consists in finding the (possibly multiple) price coefficients that satisfy the fixed point representation in expression (40). As initial price coefficients, I use a matrix whose rows correspond to different starting values for the price vector, where the only element changing across rows is the coefficient associated with the random supply, $p_{\theta}$. Due to its convergence properties, the low volatility equilibrium is straightforward to find as initial guesses using relatively low values of $p_{\theta}$ (in absolute magnitude) quickly converge to the the same fixed point price vector. 
The complications arise when trying to find the price coefficients in the unstable price vector. To find it I postulate successively decreasing (more negative) supply coefficients as an initial guesses. If the iteration brings the price coefficient to the low-volatility equilibrium, this means that the conjectured supply coefficient is still not negative enough. Beyond some threshold, the postulated value of $p_{\theta}$ is too negative and the iteration diverges. This implies that the second equilibrium value of $p_{\theta}$ must lie in between the last initial guess that produced a convergence, and the first guess that produced the divergence. I then zoom into this region, creating a new matrix of price vectors that span this narrower range of price coefficients.

I then repeat the process, every time defining a new range of price coefficients between the last converging and first diverging row vector of the initial matrix. After a few iterations, the range of values where the second equilibrium $p_{\theta}$ lies can be made arbitrarily narrow, providing an arbitrarily close approximation to the true equilibrium value. 